\title{
West African pholcid spiders: an overview, with descriptions of five new species (Araneae, Pholcidae)
}

\author{
Bernhard A. HUBER ${ }^{1} \&$ Peter KWAPONG ${ }^{2}$ \\ ${ }^{1}$ Alexander Koenig Research Museum of Zoology, Adenauerallee 160, 53113 Bonn, Germany \\ Email: b.huber@zfmk.de (corresponding author) \\ ${ }^{2}$ Department of Entomology \& Wildlife - International Stingless Bee Centre (ISBC), \\ School of Biological Sciences, University of Cape Coast, Cape Coast, Ghana \\ Email: pkwapong@yahoo.com \\ ${ }^{1}$ urn:Isid:zoobank.org:author:33607F65-19BF-4DC9-94FD-4BB88CED455F \\ ${ }^{2}$ urn:1sid:zoobank.org:author:DA9A306D-7C9B-4DF1-9529-004516F24AE7
}

\begin{abstract}
This paper summarizes current knowledge about West African pholcids. West Africa is here defined as the area south of $17^{\circ} \mathrm{N}$ and west of $5^{\circ} \mathrm{E}$, including mainly the Upper Guinean subregion of the Guineo-Congolian center of endemism. This includes all of Senegal, The Gambia, Guinea Bissau, Guinea, Sierra Leone, Liberia, Ivory Coast, Ghana, Togo and Benin. An annotated list of the 14 genera and 38 species recorded from this area is given, together with distribution maps and an identification key to genera. Five species are newly described: Anansus atewa sp. nov., Artema bunkpurugu sp. nov., Leptopholcus kintampo sp. nov., Spermophora akwamu sp. nov., and S. ziama sp. nov. The female of Quamtana kitahurira is newly described. Additional new records are given for 16 previously described species, including 33 new country records. Distribution patterns of West African pholcids are discussed, as well as possible explanations for relatively low West African pholcid species diversity as compared to Central and East Africa.
\end{abstract}

Keywords. Pholcidae, West Africa, taxonomy, identification key, diversity.

Huber B.A. \& Kwapong P. 2013. West African pholcid spiders: an overview, with descriptions of five new species (Araneae, Pholcidae). European Journal of Taxonomy 59: 1-44. http://dx.doi.org/10.5852/ejt.2013.59

\section{Introduction}

In a recent overview on African rain forest ecology and conservation (Weber et al. 2001), one of the major "strikingly similar principles" emerging from studies of "lesser known taxa" was the lack of basic information such as species lists, species distributions, and basic ecology. These "lesser known taxa" were not invertebrates though, but birds, fishes, amphibians, and reptiles (in contrast to mammals and plants). Invertebrates are barely mentioned in this otherwise superb book, probably because our knowledge of most groups is not even close to sufficient to track basic questions of rain forest ecology and conservation. While the lack of data is beyond question and may sometimes even appear hopelessly overwhelming, it is also worth to focus on the progress that has been made. The present paper adopts the latter position, concentrating on pholcid spiders and showing that many of the patterns found in plants and vertebrates are replicated in this particular taxon. 
While Pholcidae admittedly make up just a small fraction of the megadiverse spiders (about $3 \%$ of described species; Platnick 2013), our knowledge about this family has increased substantially over the last decade. Advances include alpha taxonomy (species numbers for African taxa have more than tripled since 2002, from 88 to now 287; Huber 2013, herein), but also phylogenetic data (summary in Huber 2011a), distribution patterns (e.g., Huber 2011b, 2012, 2013) and basic ecology (e.g., Huber 2009, 2013; Huber et al. 2013). Pholcidae are most diverse in well-preserved tropical forests, but they also occur in relatively arid regions (e.g., Huber 2001; Huber \& Brescovit 2003); they range from sea level to about $4000 \mathrm{~m}$ (Huber 2000, 2012); they include both widespread species with substantial dispersal capabilities (even though ballooning does not seem to occur in Pholcidae) and many small-scale endemics (Huber 2011b, 2013); and numerous taxa within the family include ecologically very different representatives (which is reflected in a wide range of body shapes and colors; cf. Figs 1-22), suggesting multiple independent evolutionary shifts among microhabitats (Dimitrov et al. 2013).

Considering the preference of Pholcidae for tropical forests, a high diversity in African rainforests was expected. Within the Guineo-Congolian center of endemism, West African forests were predicted to be less diverse than Central African forests, both for historical and current reasons (only about $12 \%$ of the original tropical moist forests remain in West Africa, as opposed to 59\% in Central Africa; Naughton-Treves \& Weber 2001). West Africa was also predicted to be less diverse than East Africa with its wider range of altitudes, its mosaic of different vegetation zones, and its highly diverse Eastern Arc. However, with pholcid material in existing collections it seemed impossible to even roughly estimate basic data like species numbers and distribution patterns. For this reason, a series of six expeditions was undertaken to East Africa (Kenya, Uganda), Central Africa (Cameroon, Gabon), and West Africa (Guinea, Ghana) in order to obtain comparable data across the African continent. The present paper summarizes the data on West Africa, complementing revisions of all major taxa in the area (Huber 2011b, 2012, 2013) and a previous summary on East African Pholcidae (Huber \& Warui 2012). The final part of this trilogy (Central Africa) is in preparation.

\section{Material and methods}

A large part of the material studied herein was collected during expeditions to Guinea (2008) and Ghana (2013). This material is currently deposited at Zoologisches Forschungsmuseum Alexander Koenig, Bonn (ZFMK). Further material was borrowed from the following institutions:

$\mathrm{AMNH}=$ American Museum of Natural History, New York

CAS = California Academy of Sciences, San Francisco

$\mathrm{MCZ}=$ Museum of Comparative Zoology, Cambridge

MNHN = Muséum national d'Histoire naturelle, Paris

MRAC $=$ Musée royal de l'Afrique Centrale, Tervuren

SMF = Forschungsinstitut und Naturmuseum Senckenberg, Frankfurt

USNM $=$ National Museum of Natural History, Washington D.C.

Methods and terminology are as in recent revisions (Huber 2011b, 2012, 2013). Measurements are in $\mathrm{mm}$ unless otherwise noted. Eye measurements are $+/-5 \mu \mathrm{m}$. Epigyna were cleared in a warm $\mathrm{NaOH}$ solution and stained with chlorazol black. Locality coordinates are in round brackets when copied from labels and original publications or when received directly from collectors, in square brackets when originating from some other source (such as online gazetteers, Google Earth, MRAC database, etc.). The following abbreviations are used:

$\mathrm{ALE}=$ anterior lateral eyes

ALS $=$ anterior lateral spinnerets

$\mathrm{AME}=$ anterior median eyes

a.s.l. = above sea level

$\mathrm{L} / \mathrm{d}=$ length/diameter

$\mathrm{PME}=$ posterior median eyes . 
HUBER B.A. \& KWAPONG P., West African pholcid spiders
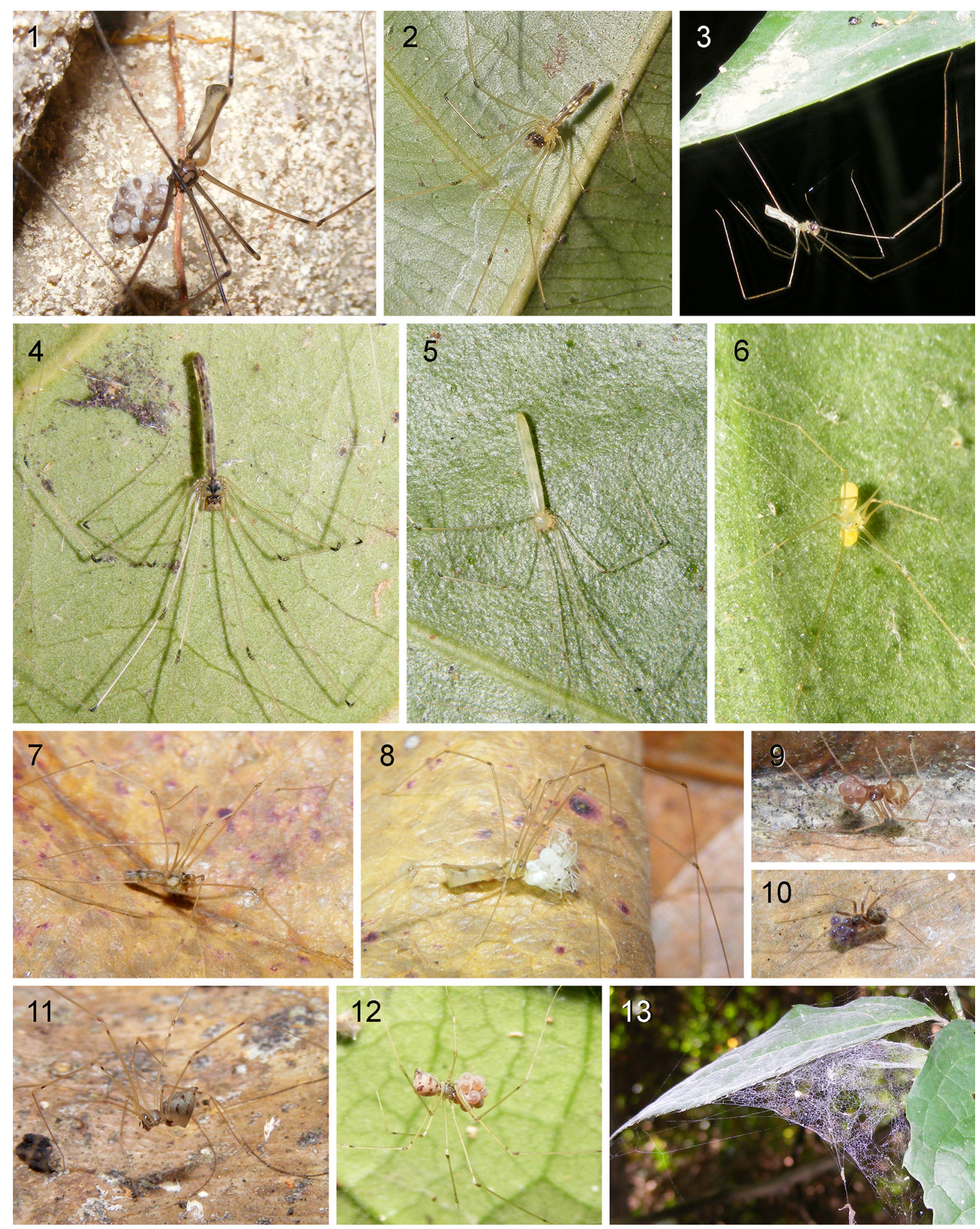

Figs 1-13. 1. Pholcus doucki Huber, 2011 from Doucki, Guinea. 2-3. Pholcus kakum Huber, 2009 from Forêt Classée de Ziama, Guinea (2) and Kakum N.P., Ghana (3). 4. Leptopholcus kintampo sp. nov. from Kintampo, Ghana. 5. Leptopholcus tipula (Simon, 1907) from Kakum N.P., Ghana. 6. Nyikoa limbe Huber, 2007 from Kakum N.P., Ghana. 7-8. Pehrforsskalia conopyga Deeleman-Reinhold \& van Harten, 2001 from Atewa, Ghana. 9-10. Anansus atewa sp. nov. from Atewa, Ghana. 11-13. Spermophora akwamu sp. nov. from Kakum N.P., Ghana (13: web). Photos BAH. 

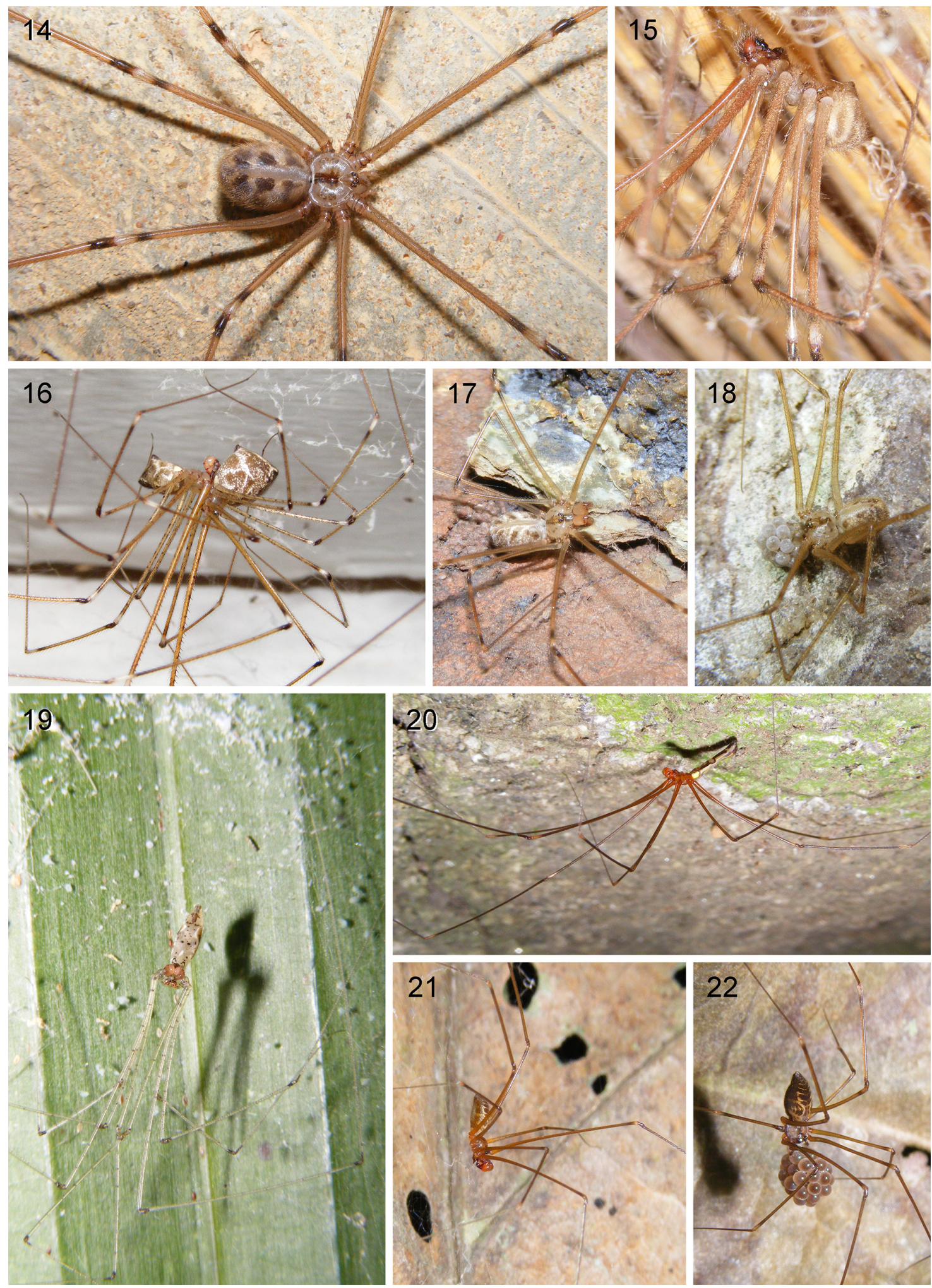

Figs 14-22. 14-15. Artema bunkpurugu sp. nov. from near Bunkpurugu (14) and from Bunkpurugu (15), Ghana. 16. Crossopriza lyoni (Blackwall, 1867) from Conakry, Guinea. 17-18. Physocyclus globosus (Taczanowski, 1874) from near Mpraeso, Ghana. 19. Smeringopus cylindrogaster (Simon, 1907) from Ankasa N.P., Ghana. 20. Smeringopina pulchra (Millot, 1941) from Kakum N.P., Ghana. 21-22. Smeringopina bomfobiri Huber, 2013 from Atewa, Ghana. Photos BAH. 
The geographic area considered here (south of $17^{\circ} \mathrm{N}$, west of $5^{\circ} \mathrm{E}$; Fig. 23) is somewhat arbitrary, especially regarding the eastern limit of "West Africa", i.e. its border to "Central Africa". In contrast to the major textbook on African spiders (Dippenaar-Schoeman \& Jocqué 1997), West Africa as defined here is largely congruent with the usage in African Rain Forest Ecology \& Conservation (Weber et al. 2001; e.g., p. 31, 119, 430). Biologically it encompasses mainly the Upper Guinean subregion of the Guineo-Congolian center of endemism (White 2001). The eastern limit of this subregion is not clear since the main current gap (Dahomey Gap; $\sim 0-3^{\circ} \mathrm{E}$ ) is not always congruent with distribution limits of taxa, which in many cases are further east (Cross River Basin of eastern Nigeria, $\sim 8^{\circ} \mathrm{E}$ ) (DowsettLemaire \& Dowsett 2001; Lawson \& Klemens 2001; Maley 2001). For the present overview, however, the exact eastern limit (western vs. eastern Nigeria) is fairly irrelevant since the pholcid fauna of Nigeria remains largely unknown.

\section{Results}

Class Arachnida Cuvier, 1812

Order Araneae Clerck, 1757

Family Pholcidae C.L. Koch, 1851

\section{Annotated list of West African genera and species}

Pholcidae described from West Africa as defined herein, with countries, references, and new records.

Anansus Huber, 2007

Anansus is widely distributed in tropical Africa (Huber 2007; Huber \& Warui 2012) with at least three species endemic to West Africa (Fig. 23).

1. Anansus aowin Huber, 2007. Ivory Coast (Huber 2007).

2. Anansus atewa sp. nov. (see page 21). Ghana.

3. Anansus ewe Huber, 2007. Ghana (Huber 2007); newly recorded for Guinea and Ivory Coast (Ivory Coast records tentative, no males available).

\section{New records}

GHANA: Central Region: 20 ภิ $\widehat{\partial} 14$ 우 3 juvs (3 vials) in ZFMK (Ar 10450-52), Kakum National Park $\left(5^{\circ} 20.9^{\prime} \mathrm{N}, 1^{\circ} 23.0^{\prime} \mathrm{W}\right), 160 \mathrm{~m}$ a.s.l., forest near entrance, day collecting, 19-20 Feb. 2013 (B.A. Huber); 2 in pure ethanol, in ZFMK (Gha 150), same data.

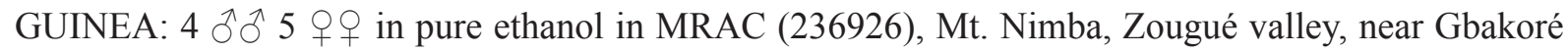
mine camp $\left[7^{\circ} 42^{\prime} \mathrm{N}, 8^{\circ} 24^{\prime} \mathrm{W}\right]$, young secondary gallery forest, canopy fogging, $780 \mathrm{~m}$ a.s.l., 5 Oct. 2011 (D. Van den Spiegel, A. Henrard); 2 q $ᄋ$ in pure ethanol in MRAC (236932), Mt. Nimba, near Gouan camp or "Station de Pompage Zié" [ $7^{\circ} 40^{\prime} \mathrm{N}, 8^{\circ} 22^{\prime} \mathrm{W}$ ], sieving of litter under "matete" (high grass), open area near road, $1250 \mathrm{~m}$ a.s.1., 11 Oct. 2011 (D. Van den Spiegel, A. Henrard).

IVORY COAST: 1 q (assigned tentatively) in MRAC (230172), Forêt de Taï, station du Centre de Recherche Ecologique (CRE) [5 50’ N, 7²1'W], sieving litter, 29 Feb. 2010 (R. Jocqué, D. Van den Spiegel); 1 q (assigned tentatively) in MRAC (230397), Adiopo Doumé, Centre Suisse de Recherche

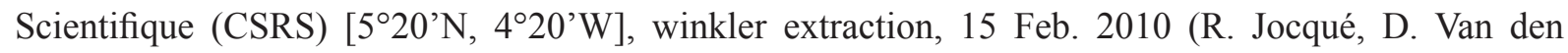
Spiegel). 


\section{Artema Walckenaer, 1837}

The natural distribution of Artema ranges from the Sudanian region of West Africa (Fig. 24) to Central Asia. Millot (1941) was the first to record the genus from West Africa, and he noted that most of his material of "A. mauriciana Walckenaer, 1837" (=A. atlanta Walckenaer, 1837) did not agree well with previous illustrations of this pantropical species. After comparing numerous West African specimens with $A$. atlanta from all over the World, we agree on these substantial morphological differences and formally describe the West African species below.
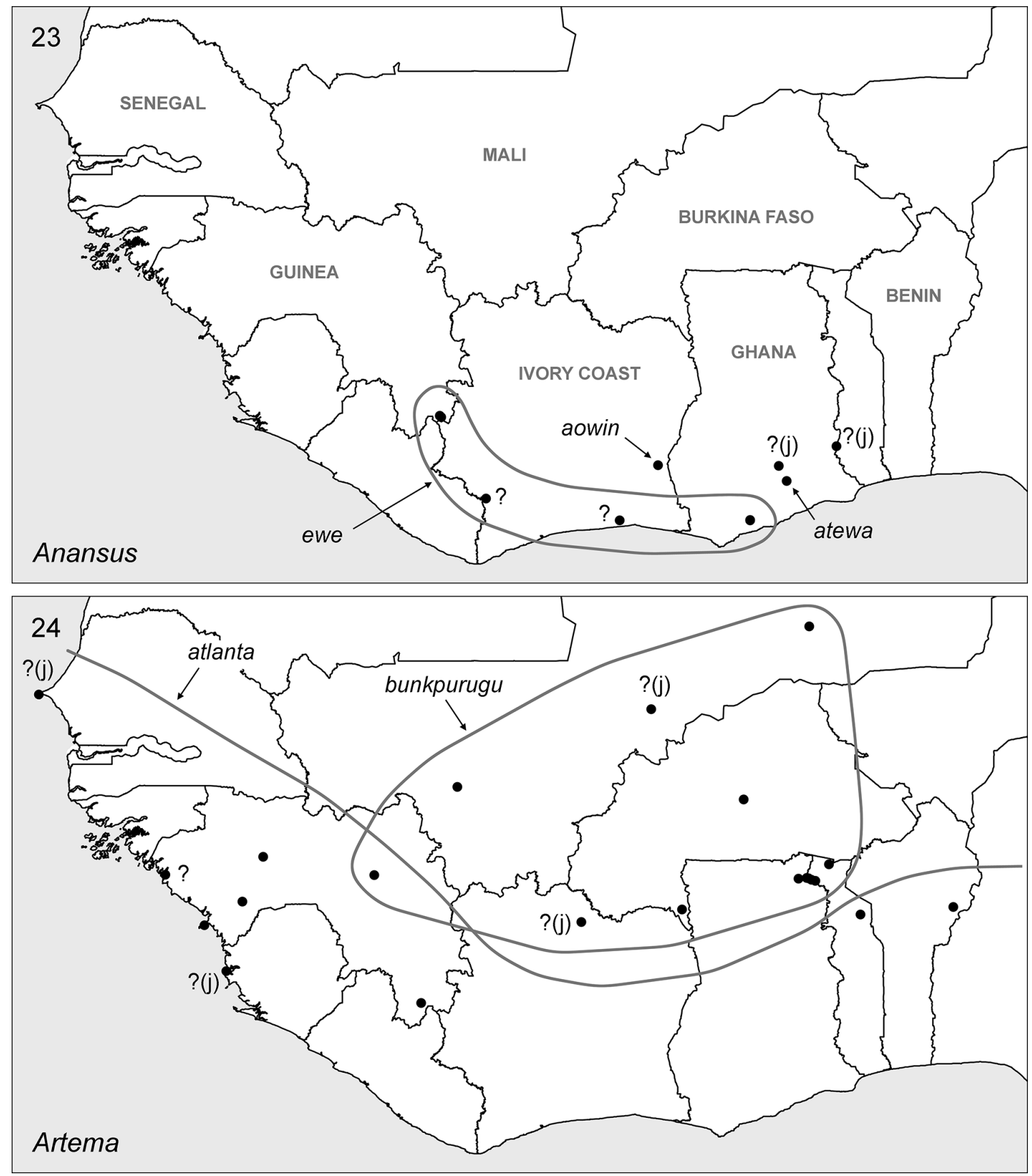

Figs 23-24. Known distributions of the genera Anansus Huber, 2007 and Artema Walckenaer, 1837 in West Africa. Question marks denote uncertain identifications (Anansus ewe: two localities with females only) or impossible identifications [“?(j)": juveniles only]. 
4. Artema atlanta Walckenaer, 1837. Guinea (Millot 1941: only the variant male from Kouroussa); newly recorded for Togo and Benin.

\section{New records}

GUINEA: $10^{\top}$ in pure ethanol, in ZFMK (Gui 82), Conakry, in building $\left(9^{\circ} 31^{\prime} \mathrm{N}, 13^{\circ} 43^{\prime} \mathrm{W}\right), \sim 30 \mathrm{~m}$ a.s.1., 18 Nov. 2008 (B.A. Huber). Guinée Forestière: 1 q in ZFMK (Ar 10453), Nzérékoré ( $7^{\circ} 45.3^{\prime} \mathrm{N}$, $\left.8^{\circ} 49.2^{\prime} \mathrm{W}\right), 480 \mathrm{~m}$ a.s.1., in building, 28 Nov. 2008 (B.A. Huber). Moyenne-Guinée: $1 \delta^{\Uparrow}$ in pure ethanol, in ZFMK (Gui 111), Pita, at building ( $\left(11^{\circ} 03.6^{\prime} \mathrm{N}, 12^{\circ} 23.7^{\prime} \mathrm{W}\right), 1010 \mathrm{~m}$ a.s.1., 25 Nov. 2008 (B.A. Huber). Kankan: $1 \delta^{\wedge}$ in MNHN, Kouroussa [10³9'N, 953’W], Aug. 1937 (J. Millot) [this is probably Millot's (1941) variant male of his "Artema mauriciana"].

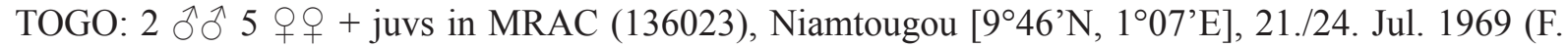
Puylaert).

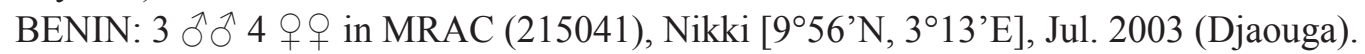

\section{Note}

Millot's (1941) specimens from Kindia, Guinea (2 juvs; 9 missing), Ferkessedougou, Ivory Coast (1 juv.), and Gao, Mali (2 juvs) are in MNHN but juveniles of A. atlanta and A. bunkpurugu sp. nov. are indistinguishable so their identity is unclear. The same is true for juvenile specimens from Dakar, Senegal in AMNH, from Freetown, Sierra Leone in SMF, and from Bandiagara (70 km E Mopti), Mali in CAS.

5. Artema bunkpurugu sp. nov. (see page 24). Ghana, Burkina Faso, Mali, Guinea, Togo.

\section{Crossopriza Simon, 1893}

Apart from the pantropical synanthropic C. lyoni (Blackwall, 1867), only C. soudanensis Millot, 1941 is known to occur in West Africa (Fig. 25).

6. Crossopriza lyoni (Blackwall, 1867). Mali, Nigeria (Huber et al. 1999); newly recorded for Senegal, The Gambia, Guinea, Sierra Leone, Ivory Coast, Ghana, Burkina Faso, Benin, and Niger.

\section{New records}

SENEGAL: Kaolack Prov.: 1 q 1 juv. in ZFMK (Ar 5207), Sonkoron, Kaymor Region [ $\sim 13^{\circ} 47^{\prime} \mathrm{N}$, 15³3’W], Jul. 1993 (M. Sarr.).

THE GAMBIA: 3 우 9 in ZFMK (Ar 5404), Banjul $\left(13^{\circ} 27.6^{\prime} \mathrm{N}, 16^{\circ} 34.7^{\prime} \mathrm{W}\right)$, on wall, 20 Oct. 2008

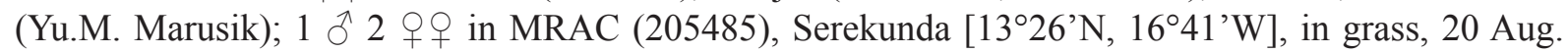
1996 (G. Beyens).

GUINEA: $1 \bigcirc^{\wedge} 2$ 우 $ᄋ$ in pure ethanol in ZFMK (Gui 103), Conakry, in building ( $9^{\circ} 34.1^{\prime} \mathrm{N}, 13^{\circ} 39.7^{\prime} \mathrm{W}$ ), 40 m a.s.1., 18 Nov. 2008 (B.A. Huber). Guinée Forestière: 1 đo in ZFMK (Ar 10454), Nzérékoré

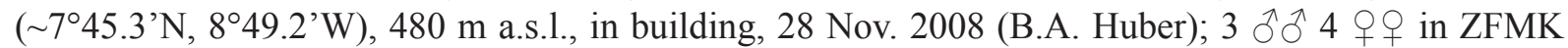
(Ar 10455), Kissidougou, in building $\left(9^{\circ} 11.2^{\prime} \mathrm{N}, 10^{\circ} 05.6^{\prime} \mathrm{W}\right), 540 \mathrm{~m}$ a.s.l., 26 Nov. 2008 (B.A. Huber); 2 우 3 juvs in pure ethanol, in ZFMK (Gui 90), same data. Basse-Guinée: 1 đ 1 ㅇ in ZFMK (Ar 10456), Kindia, in building ( $\left.10^{\circ} 03^{\prime} \mathrm{N}, 12^{\circ} 51^{\prime} \mathrm{W}\right), 400 \mathrm{~m}$ a.s.1., 20 Nov. 2008 (B.A. Huber).

SIERRA LEONE: 1 q in MRAC (174657), Makeni [8 ${ }^{\circ} 53^{\prime} \mathrm{N}, 12^{\circ} 03^{\prime} \mathrm{W}$ ], “maison”, 19 Jan. 1993 (F. Rensonnet).

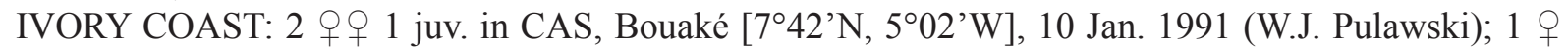
in SMF, Comoé National Park, Research Station Univ. of Würzburg [ 9.1 $1^{\circ} \mathrm{N}, 3.7^{\circ} \mathrm{W}$ ], 23 Sep. 2002 (S.

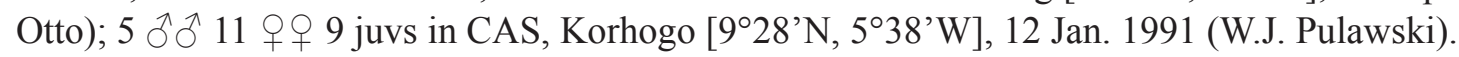

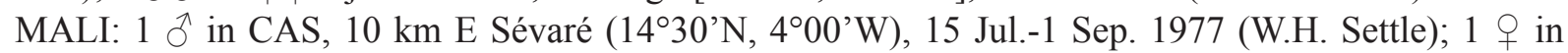

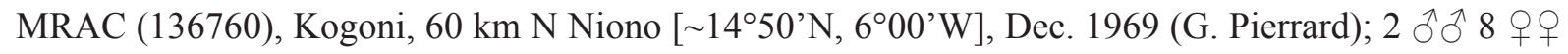


in MRAC (136761, 138808), M'Pesoba [Mpessoba, 1240’N, 543’W], Sep.-Oct. 1969 and Sep. 1970 (G. Pierrard).

GHANA: Northern Region: 1 i in ZFMK (Ar 10457), Bunkpurugu (10³1.1 'N, 0 0 05.5'E), $230 \mathrm{~m}$ a.s.l., in buildings, 7 Mar. 2013 (B.A. Huber); 101 in pure ethanol, in ZFMK (Gha 127), same data; 5 우우 in ZFMK (Ar 10458), Bunkpurugu $\left(10^{\circ} 31.3^{\prime} \mathrm{N}, 0^{\circ} 05.8^{\prime} \mathrm{E}\right), 250 \mathrm{~m}$ a.s.l., in building, $6 \mathrm{Mar}$. 2013

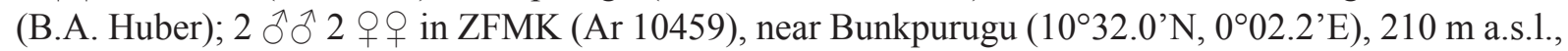
under concrete bridge, 8 Mar. 2013 (B.A. Huber); 1 1 juv. in pure ethanol, in ZFMK (Gha 142), same data; $1 \delta^{\wedge} 3$ 우 in ZFMK (Ar 10460), near Bimbaga $\left(10^{\circ} 34.5^{\prime} \mathrm{N}, 0^{\circ} 03.8^{\prime} \mathrm{W}\right), 330 \mathrm{~m}$ a.s.l., under concrete bridge, 8 Mar. 2013 (B.A. Huber); 1 + 2 juvs in ZFMK (Ar 10461), near Gambaga (10³1.9'N, $0^{\circ} 25.3^{\prime} \mathrm{W}$ ), $350 \mathrm{~m}$ a.s.1., under concrete bridge, 8 Mar. 2013 (B.A. Huber); 1 के in ZFMK (Ar 10462), near Gambaga $\left(10^{\circ} 29.3^{\prime} \mathrm{N}, 0^{\circ} 28.9^{\prime} \mathrm{W}\right), 300 \mathrm{~m}$ a.s.l., under concrete bridge, 8 Mar. 2013 (B.A. Huber); 1 ㅇ in ZFMK (Ar 10463), Tamale ( $\left.9^{\circ} 24.4^{\prime} \mathrm{N}, 0^{\circ} 50.4^{\prime} \mathrm{W}\right), 195 \mathrm{~m}$ a.s.1., in building, 5 Mar. 2013 (B.A. Huber). Greater Accra Region: 1 \% in ZFMK (Ar 10464), Accra ( $\left.5^{\circ} 34.4^{\prime} \mathrm{N}, 0^{\circ} 13.1^{\prime} \mathrm{W}\right), 15 \mathrm{~m}$ a.s.1., in building, 1 Mar. 2013 (B.A. Huber). Ashanti Region: $1 \overbrace{}^{\wedge} 1$ \& in ZFMK (Ar 10465), Kumasi (6²3.4’ N, $1^{\circ} 38.1^{\prime} \mathrm{W}$ ), $310 \mathrm{~m}$ a.s.1., in building, 4 Mar. 2013 (B.A. Huber). Brong-Ahafo Region: 1 ㅇ in ZFMK (Ar 10466), Techiman $\left(7^{\circ} 34.5^{\prime} \mathrm{N}, 1^{\circ} 57.5^{\prime} \mathrm{W}\right), 420 \mathrm{~m}$ a.s.1., in building, 4 Mar. 2013 (B.A. Huber). Eastern Region: 2 우 in ZFMK (Ar 10467), Suhum ( $\left.6^{\circ} 02.4^{\prime} \mathrm{N}, 0^{\circ} 27.1^{\prime} \mathrm{W}\right), 215 \mathrm{~m}$ a.s.l., in building, 10 Mar. 2013 (B.A. Huber).

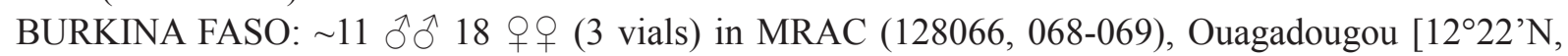
1³1'W], Apr.-May 1965 (B. Roman); 1 ô in MCZ (34049), same locality, "bathroom wall", 16 Jul. 1977 (Brinckerhoff).

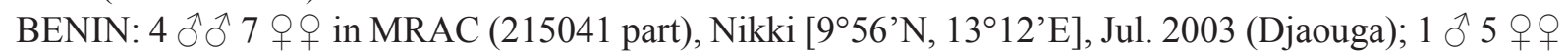
in MRAC (212766 part), Adjohoun [6² $\left.{ }^{\circ} \mathrm{N}, 2^{\circ} 30^{\prime} \mathrm{E}\right]$, in house, 23 Dec. 2002 (S. Tchibozo).

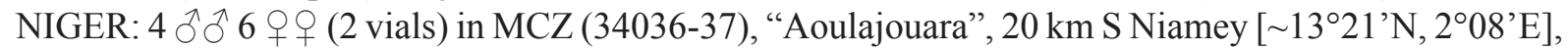

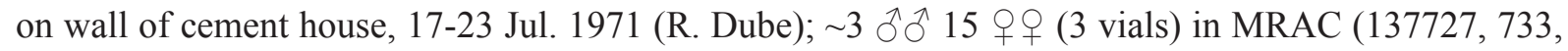
734), Niamey [ $\left.13^{\circ} 30^{\prime} \mathrm{N}, 2^{\circ} 07^{\prime} \mathrm{E}\right]$, Sep.-Oct. 1970 (E. van Impe).

NIGERIA: Osun: 1 ㅇ in MRAC (174602 part), Ile-Ife, Obafemi Awolowo Univ. campus [7²8'N, 434’E], 1991 (H. Segers).

7. Crossopriza soudanensis Millot, 1941. Mali, Burkina Faso (Millot 1941).

Leptopholcus Simon, 1893

Of the ten species of Leptopholcus currently known from mainland Africa (Huber 2011b; herein), two are endemic to West Africa (L. guineensis Millot, 1941; L. kintampo sp. nov.); the third [L. tipula (Simon, 1907)] occurs throughout the entire Guineo-Congolian rainforest (Huber 2011b) (Fig. 26).

8. Leptopholcus guineensis Millot, 1941. Senegal, The Gambia, Guinea (Millot 1941; Huber 2009); newly recorded for Ghana.

\section{New records}

GUINEA: 7 đิ $\widehat{0} 5$ 우 in pure ethanol, in MRAC (236931), Mt. Nimba, Forêt de Zié, near Gouan camp

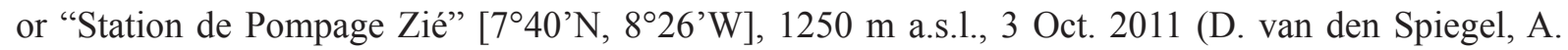
Henrard); 1 in pure ethanol, in MRAC (236927 part), Mt Nimba, Zougué valley, near Gbakoré mine camp [ $7^{\circ} 42^{\prime} \mathrm{N}, 8^{\circ} 24^{\prime} \mathrm{W}$ ], young secondary gallery forest, canopy fogging, $780 \mathrm{~m}$ a.s.1., 5 Oct. 2011 (D. Van den Spiegel, A. Henrard).

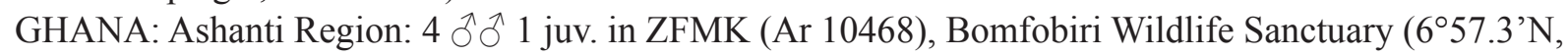
$\left.1^{\circ} 11.1^{\prime} \mathrm{W}\right), 160 \mathrm{~m}$ a.s.l., forest near river, 3 Mar. 2013 (B.A. Huber); 1 \& 3 juvs in pure ethanol, in ZFMK (Gha 131), same data. Eastern Region: $1 \overbrace{}^{\Uparrow} 1$ juv. in ZFMK (Ar 10469), near Mpraeso ( $6^{\circ} 34.1^{\prime} \mathrm{N}$, $0^{\circ} 43.9^{\prime} \mathrm{W}$ ), $370 \mathrm{~m}$ a.s.1., degraded forest near road, 10 Mar. 2013 (B.A. Huber); 2 juvs in pure ethanol, in 
ZFMK (Gha 152), same data. Volta Region: 2 $\widehat{\jmath} 4 q q 7$ juvs in ZFMK (Ar 10470), Agumatsa Wildlife Sanctuary, Wli waterfall $\left(7^{\circ} 06.2^{\prime} \mathrm{N}, 0^{\circ} 36.0^{\prime} \mathrm{E}\right), \sim 300 \mathrm{~m}$ a.s.1., forest near waterfall, $27 \mathrm{Feb} .2013$ (B.A. Huber); 5 juvs in pure ethanol, in ZFMK (Gha 164), same data; 1 o 1 q 1 juv. in ZFMK (Ar 10471), Tagbo waterfall $\left(7^{\circ} 00.7^{\prime} \mathrm{N}, 0^{\circ} 34.4^{\prime} \mathrm{E}\right), \sim 500 \mathrm{~m}$ a.s.l., forest near waterfall, 28 Feb. 2013 (B.A. Huber). Central Region: 1 i in MRAC (217306), Kakum Forest, primary forest, 11 Nov. 2005 (R. Jocqué, D. de Bakker, L. Baert).
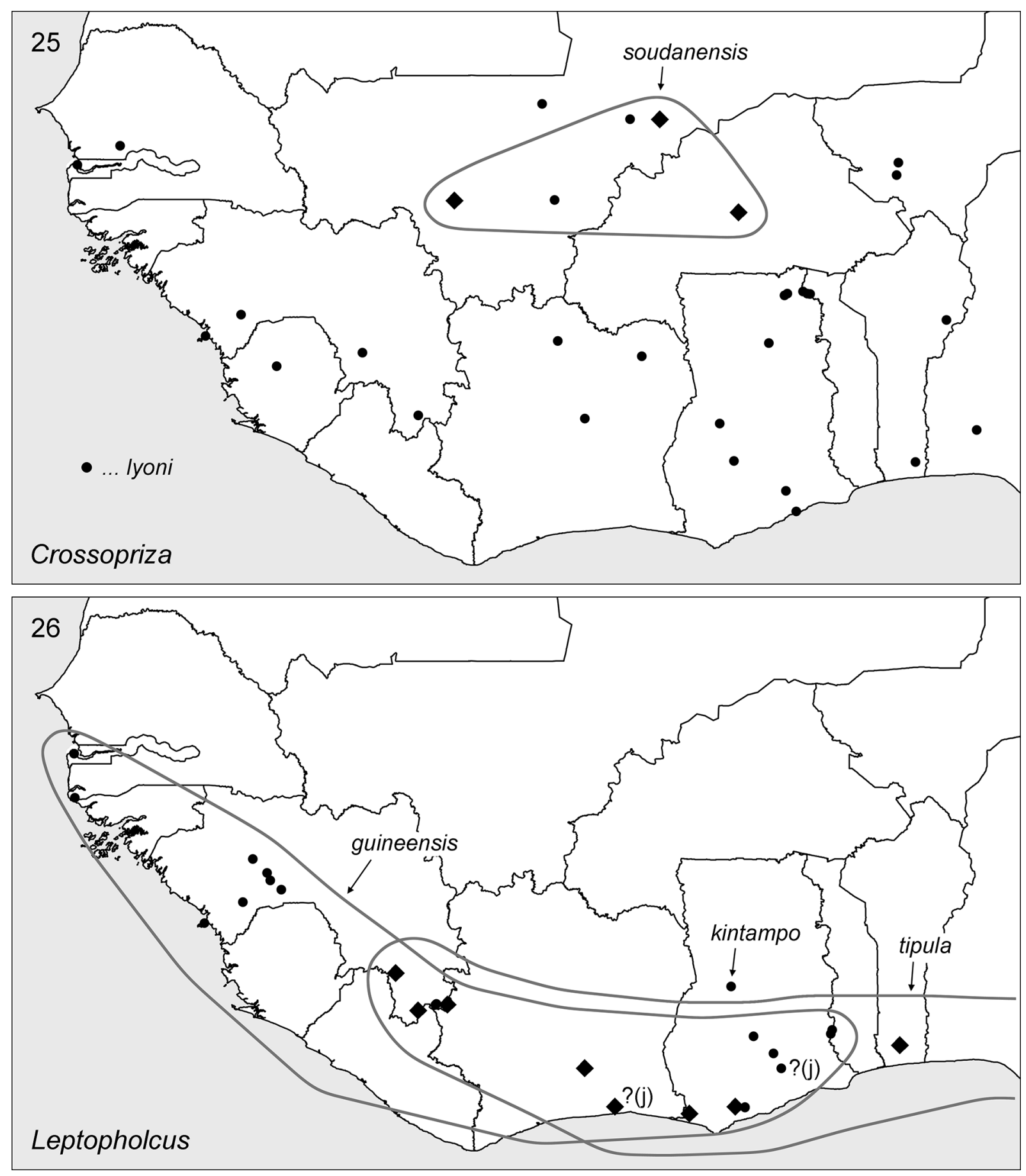

Figs 25-26. Known distributions of the genera Crossopriza Simon, 1893 and Leptopholcus Simon, 1893 in West Africa. Question marks denote uncertain identifications [“?(j)": juveniles only]. 
9. Leptopholcus kintampo sp. nov. (see page 28). Ghana.

10. Leptopholcus tipula (Simon, 1907). Guinea, Ghana, Benin (Huber 2009, 2011b); newly recorded for Ivory Coast.

\section{New records}

IVORY COAST: 1 i in MNHN, Lamto [6¹3’N, 502’W?], 26 Aug. 1963 (collector not given); 1 juv. in MRAC (230358 part), Adiopo Doumé, Centre Suisse de Recherche Scientifique (CSRS) [5²0'N, $\left.4^{\circ} 20^{\prime} \mathrm{W}\right]$, small dry forest, beating, 25 Feb. 2010 (D. van den Spiegel, R. Jocqué).

GHANA: Central Region: 1 i in pure ethanol in ZFMK (Gha 143), Kakum National Park ( $5^{\circ} 20.9^{\prime} \mathrm{N}$, $\left.1^{\circ} 23.0^{\prime} \mathrm{W}\right), 160 \mathrm{~m}$ a.s.1., forest near entrance, day collecting, 20 Feb. 2013 (B.A. Huber). Western

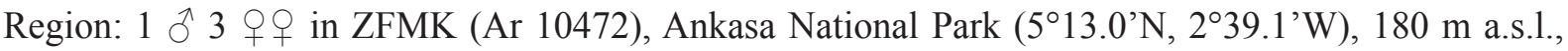
forest near entrance, night collecting, 22 Feb. 2013 (B.A. Huber); 1 \& 3 juvs in pure ethanol, in ZFMK (Gha 156), same data.

Micropholcus Deeleman-Reinhold \& Prinsen, 1987

Only the synanthropic M. fauroti (Simon, 1887) occurs in West Africa (Huber 2011b) (Fig. 27).

11. Micropholcus fauroti (Simon, 1887). Senegal, The Gambia, Sierra Leone, Guinea (Millot 1941; Huber 2011b); newly recorded for Ivory Coast and Ghana.

\section{New records}

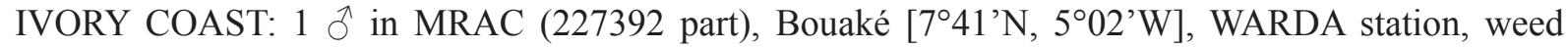
management experiment, upland rice, 13 Aug. 1995 (A. Russell-Smith).

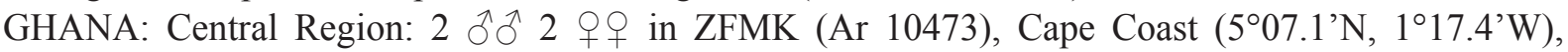
$15 \mathrm{~m}$ a.s.1., in building, 19 Feb. 2013 (B.A. Huber); 1 \& in ZFMK (Ar 10474), Cape Coast ( $5^{\circ} 06.3^{\prime} \mathrm{N}$, $\left.1^{\circ} 14.8^{\prime} \mathrm{W}\right), 15 \mathrm{~m}$ a.s.l., in building, 23 Feb. 2013 (B.A. Huber). Greater Accra Region: 1 ○ 1 q in ZFMK (Ar 10475), Accra ( $\left.5^{\circ} 34.4^{\prime} \mathrm{N}, 0^{\circ} 15.2^{\prime} \mathrm{W}\right), 45 \mathrm{~m}$ a.s.l., in building, $17 \mathrm{Feb} .2013$ (B.A. Huber). Ashanti

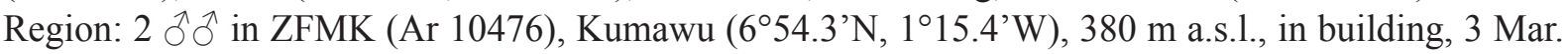

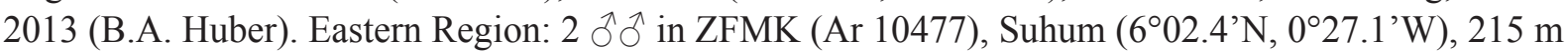
a.s.1., in building, 10 Mar. 2013 (B.A. Huber). Volta Region: 1 ภ 1 q in ZFMK (Ar 10478), Hohoe ( $\left.7^{\circ} 09.3^{\prime} \mathrm{N}, 0^{\circ} 29.5^{\prime} \mathrm{E}\right), 200 \mathrm{~m}$ a.s.l., in building, $27 \mathrm{Feb} .2013$ (B.A. Huber).

Modisimus Simon, 1893

The pantropical M. culicinus (Simon, 1893) is the only representative of this otherwise New World genus in Africa. It is probably not rare but due to its small size and cryptic lifestyle it has previously been recorded only twice from Africa (Congo DR and Kenya; Lessert 1938 - sub Hedypsilus lawrencei Lessert, 1938 - and Huber \& Warui 2012). It is here newly recorded for West Africa (Fig. 28).

12. Modisimus culicinus (Simon, 1893). Newly recorded for West Africa (Sierra Leone).

\section{New record}

SIERRA LEONE: $1 \sigma^{`}$ in MRAC (148459 part), Freetown, Mt. Aureol [8 $\left.8^{\circ} 28.7^{\prime} N, 13^{\circ} 13.3^{\prime} \mathrm{W}\right]$, Sep. 1976 (D. Olu-Pitt).

\section{Nyikoa Huber, 2007}

Nyikoa is a monotypic genus with the leaf-dwelling N. limbe Huber, 2007 covering the entire GuineoCongolian rainforest (Huber 2007, 2009). 
13. Nyikoa limbe Huber, 2007. Ghana, Guinea (Huber 2007, 2009); newly recorded for Ivory Coast.

\section{New records}

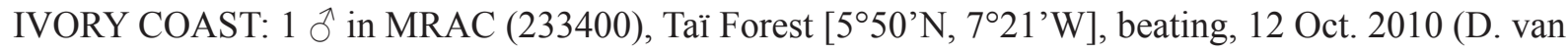
den Spiegel, A. Kablan).

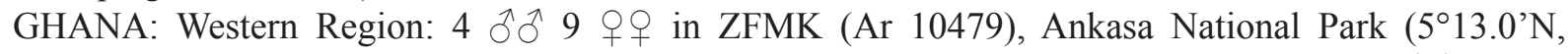

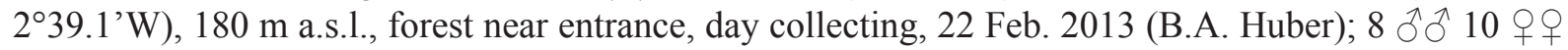
in ZFMK (Ar 10480), same data but night collecting; 1 त 1 q in pure ethanol, in ZFMK (Gha 155),
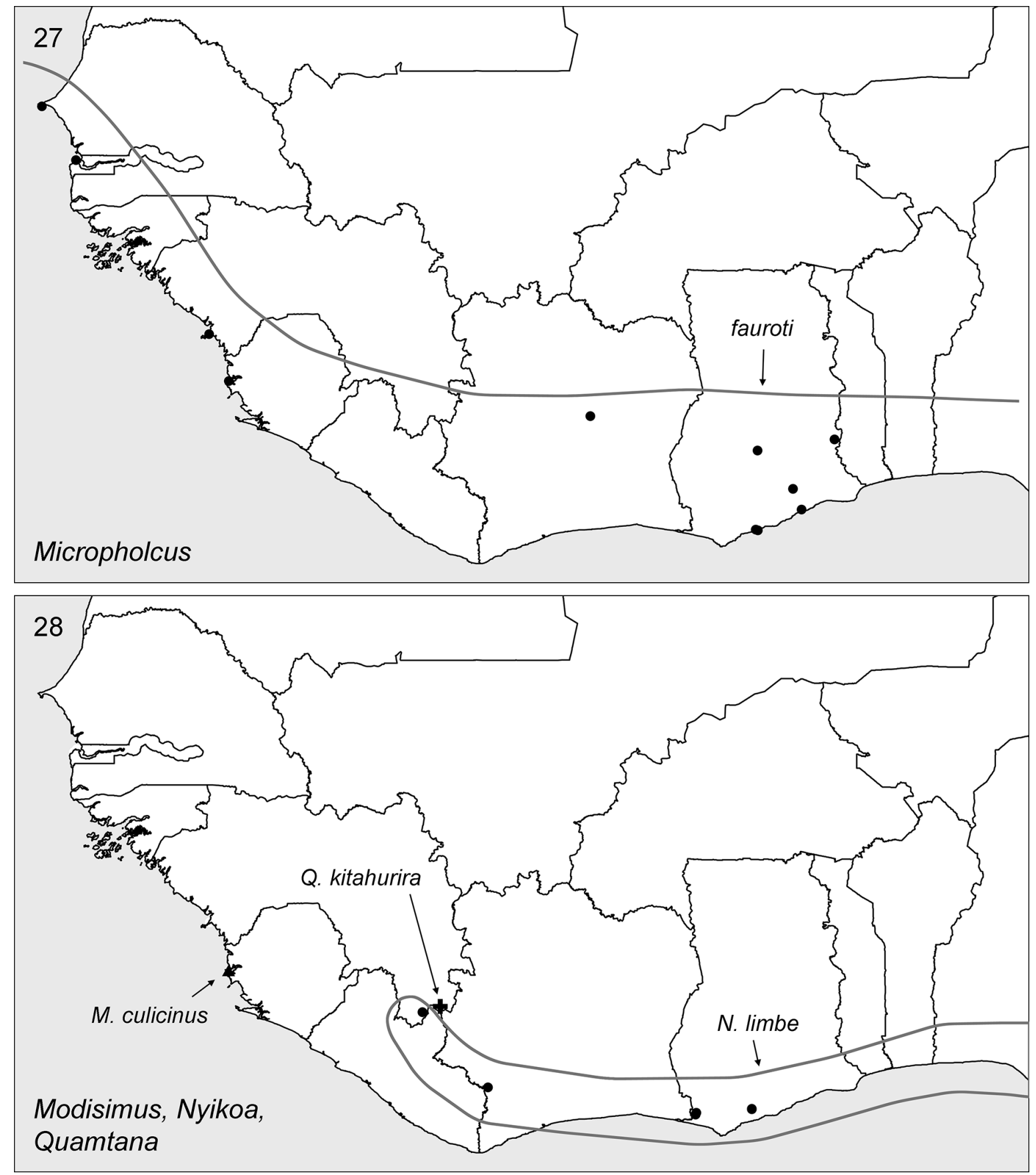

Figs 27-28. Known distributions of the genera Micropholcus Deeleman-Reinhold \& Prinsen, 1987, Modisimus Simon, 1893, Nyikoa Huber, 2007, and Quamtana Huber, 2003 in West Africa. 
same data; 1 đ in ZFMK (Ar 10481), Ankasa National Park, forest along Big Tree Trail $\left(\sim 5^{\circ} 15.1{ }^{\prime} \mathrm{N}\right.$, $2^{\circ} 38.4^{\prime} \mathrm{W}$ ), $\sim 100 \mathrm{~m}$ a.s.1., 23 Feb. 2013 (B.A. Huber). Central Region: 6 ○े $\delta^{\lambda} 8$ 우 (2 vials) in ZFMK (Ar 10482-83), Kakum National Park $\left(5^{\circ} 20.9^{\prime} \mathrm{N}, 1^{\circ} 23.0^{\prime} \mathrm{W}\right), 160 \mathrm{~m}$ a.s.1., forest near entrance, day collecting, 19-20 Feb. 2013 (B.A. Huber); 1 đ 1 क in ZFMK (Ar 10484), same data but night collecting; 1 \ 2 우 in pure ethanol, in ZFMK (Gha 146), same data.

Pehrforsskalia Deeleman-Reinhold \& van Harten, 2001

Pehrforsskalia includes three described species (Huber 2011b). Only the widely distributed P. conopyga Deeleman-Reinhold \& van Harten, 2001 occurs in West Africa (Fig. 29).

14. Pehrforsskalia conopyga Deeleman-Reinhold \& van Harten, 2001. Guinea, Sierra Leone, Ivory Coast, Nigeria (Deeleman-Reinhold \& van Harten 2001; Huber 2009); newly recorded for The Gambia and Ghana.

\section{New records}

THE GAMBIA: 2 우 in ZFMK, Banjul, Abuko Nature Reserve $\left(13^{\circ} 23.5^{\prime} \mathrm{N}, 16^{\circ} 39.0^{\prime} \mathrm{W}\right)$, primary gallery forest, 17 Oct. 2008 (Yu.M. Marusik).

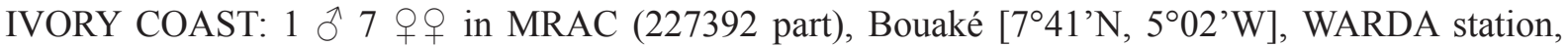
weed management experiment, upland rice, 13 Aug. 1995 (A. Russell-Smith); 5 đo 2 우 in MRAC (225855), Gagnoa [ $6^{\circ} 08^{\prime} \mathrm{N}, 5^{\circ} 57^{\prime} \mathrm{W}$ ], upland rice, 10 Apr. 1995 (A. Russell-Smith); 1 ○ 2 우 in ZFMK (Ar 5171), Bobayo near Gagnoa [601.6’ N, 601.9’W], in upland rice, 26 Aug. 1992 (A. Russell-Smith); 1 đ 1 2 juvs in ZFMK (Ar 5170), “Kroutla” nr. Oumé [ $\left.\sim 6^{\circ} 23^{\prime} \mathrm{N}, 5^{\circ} 25^{\prime} \mathrm{W}\right], 26$ Aug. 1992 (A. RussellSmith).

GHANA: Eastern Region: 1 đ 3 qq in ZFMK (Ar 10485), Atewa Hills, Atewa Atwirebu Reserve $\left(6^{\circ} 13.8^{\prime} \mathrm{N}, 0^{\circ} 33.5^{\prime} \mathrm{W}\right), 740 \mathrm{~m}$ a.s.1., 25 Feb. 2013 (B.A. Huber); 2 우 1 juv. in pure ethanol, in ZFMK (Gha 136), same data; 1 ^ 1 i in ZFMK (Ar 10486), Atewa Hills, Atewa Atwirebu Reserve $\left(6^{\circ} 13.8^{\prime} \mathrm{N}\right.$, $\left.0^{\circ} 32.4^{\prime} \mathrm{W}\right), 500 \mathrm{~m}$ a.s.1., degraded forest along road, $24 \mathrm{Feb} .2013$ (B.A. Huber); 1 17 우 in ZFMK (Ar 10487), near Mpraeso ( $\left.6^{\circ} 34.1^{\prime} \mathrm{N}, 0^{\circ} 43.9^{\prime} \mathrm{W}\right), 370 \mathrm{~m}$ a.s.l., degraded forest near road, $10 \mathrm{Mar} .2013$

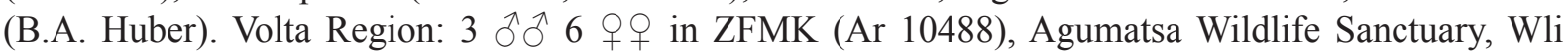
waterfall $\left(7^{\circ} 06.2^{\prime} \mathrm{N}, 0^{\circ} 36.0^{\prime} \mathrm{E}\right), \sim 300 \mathrm{~m}$ a.s.l., forest near waterfall, $27 \mathrm{Feb} .2013$ (B.A. Huber); 2 우 in pure ethanol, in ZFMK (Gha 163), same data; 5 ऽㅎํ 4 우우 in ZFMK (Ar 10489), Tagbo waterfall $\left(7^{\circ} 00.7^{\prime} \mathrm{N}, 0^{\circ} 34.4^{\prime} \mathrm{E}\right), \sim 500 \mathrm{~m}$ a.s.1., forest near waterfall, 28 Feb. 2013 (B.A. Huber). Ashanti Region: 1 오 4 우우 in ZFMK (Ar 10490), Bomfobiri Wildlife Sanctuary (6 $\left.6^{\circ} 57.3^{\prime} \mathrm{N}, 1^{\circ} 11.1^{\prime} \mathrm{W}\right), 160 \mathrm{~m}$ a.s.1., forest near river, 3 Mar. 2013 (B.A. Huber); 1 1 juv. in pure ethanol, in ZFMK (Gha 132), same data.

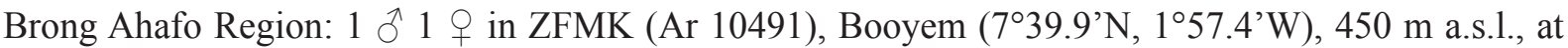
large rocks, 4 Mar. 2013 (B.A. Huber); 1 ○̊ 2 우 in ZFMK (Ar 10492), Kintampo Falls (805.3'N, $\left.1^{\circ} 41.9^{\prime} \mathrm{W}\right), 280 \mathrm{~m}$ a.s.1., degraded forest along stream, 5 Mar. 2013 (B.A. Huber).

\section{Pholcus Walckenaer, 1805}

In the area considered here, Pholcus is mainly represented by the five species of the West African endemic guineensis group (Huber 2011b; Fig. 30). Pholcus berlandi Millot, 1941 from Senegal seems to belong in a species group more diverse in northern Africa (Huber 2011b); Pholcus kakum Huber, 2009 covers the entire Guineo-Congolian rainforest (Huber 2011b).

15. Pholcus berlandi Millot, 1941. Senegal (Millot 1941).

16. Pholcus bourgini Millot, 1941. Guinea (Millot 1941; Huber 2011b).

17. Pholcus chattoni Huber, 2011. Guinea, Ivory Coast (Millot 1941; Huber 2011b). 


\section{New records}

GUINEA: $1 \partial^{\rtimes} 1$ i in pure ethanol, in MRAC (236923), Mt. Nimba, Château [740’N, $8^{\circ} 23^{\prime} \mathrm{W}$ ], dry mine adit, on wall, 10 Oct. 2011 (D. Van den Spiegel, A. Henrard); 3 우 in pure ethanol, in MRAC (236924), Mt. Nimba, Pierre Richaud, entrance of disaffected humid mine, adit, on beams, 7 Oct. 2011 (D. Van den Spiegel, A. Henrard).
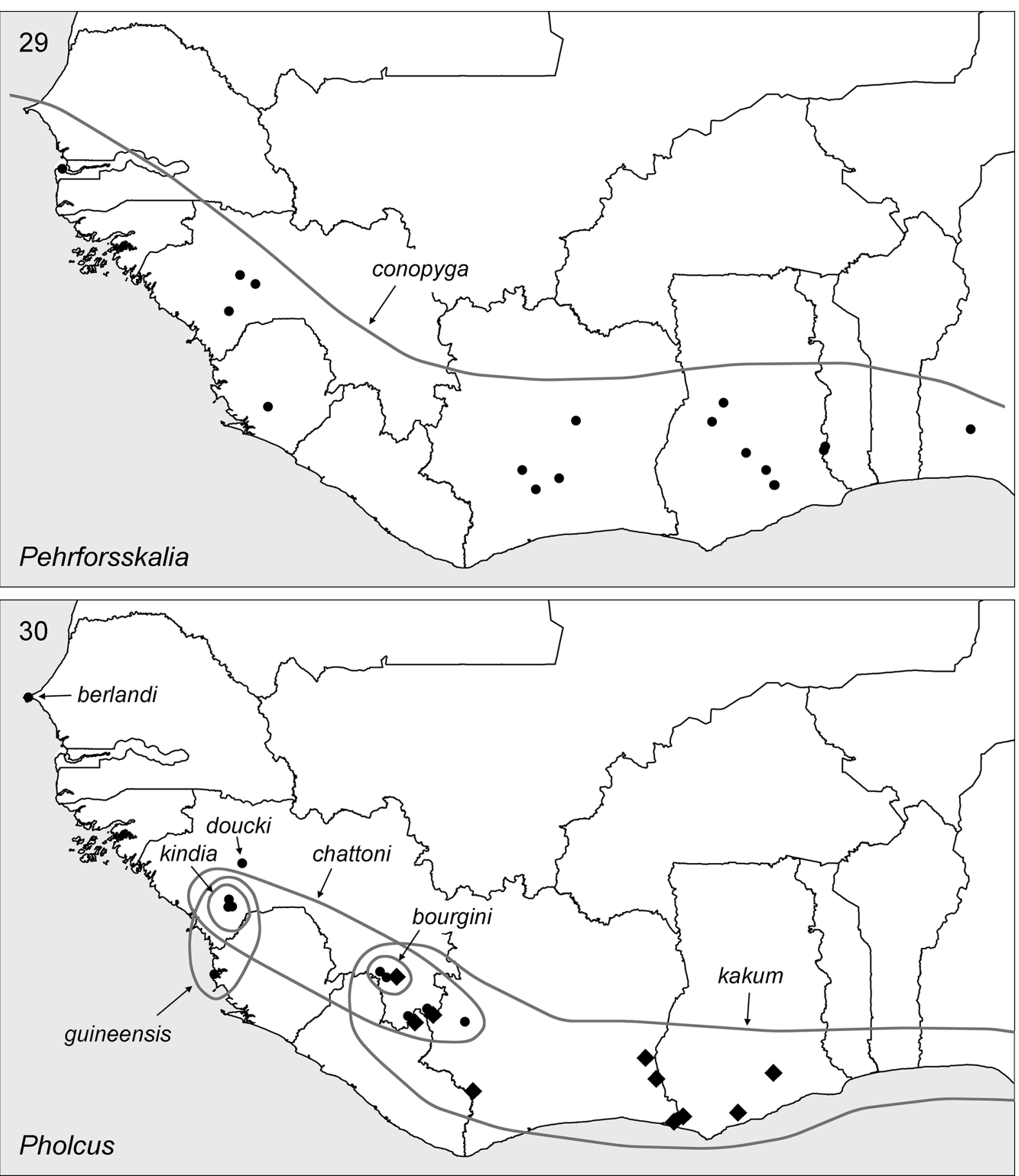

Figs 29-30. Known distributions of the genera Pehrforsskalia Deeleman-Reinhold \& van Harten, 2001 and Pholcus Walckenaer, 1805 in West Africa. 
18. Pholcus doucki Huber, 2011. Guinea (Huber 2011b).

19. Pholcus guineensis Millot, 1941. Guinea, Sierra Leone (Millot 1941; Huber 2011b).

20. Pholcus kakum Huber, 2009. Guinea, Ivory Coast, Ghana (Huber 2009).

\section{New records}

GUINEA: 1 1 juv. in pure ethanol, in MRAC (236927 part), Mt Nimba, Zougué valley, near Gbakoré mine camp [ $7^{\circ} 42^{\prime} \mathrm{N}, 8^{\circ} 24^{\prime} \mathrm{W}$ ], young secondary gallery forest, canopy fogging, $780 \mathrm{~m}$ a.s.1., 5 Oct. 2011 (D. Van den Spiegel, A. Henrard).

IVORY COAST: 1 i in MRAC (230510), Taï Forest, Centre de Recherche Ecologique (CRE) [550’N, $7^{\circ} 21^{\prime} \mathrm{W}$ ], beating in forest near Chimpanzee Camp, 22 Feb. 2010 (R. Jocqué, M. Diarassouba); 4 + 9 in MRAC (230295), same locality but forest across river, forest on clayey soil, beating, 20 Feb. 2010; 1 ㅇ in MRAC (230245), same locality but forest E of camp, "layon Gérard", inundated forest, sieved litter, 20 Feb. 2010 (R. Jocqué, L. Oulaï); 1 đ in MRAC (233345), Taï Forest, beating, 1 Sep. 2010 (D. Van

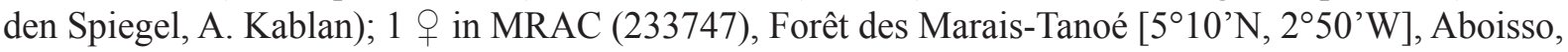
Dohouan, beating, 25 Oct. 2010 (A. Kablan).

GHANA: Central Region: 5 की 8 우우 (2 vials) in ZFMK (Ar 10493-94), Kakum National Park $\left(5^{\circ} 20.9^{\prime} \mathrm{N}, 1^{\circ} 23.0^{\prime} \mathrm{W}\right), 160 \mathrm{~m}$ a.s.l., forest near entrance, day collecting, 19-20 Feb. 2013 (B.A. Huber); 1 o in ZFMK (Ar 10495), same data but night collecting; 2 우 in pure ethanol, in ZFMK (Gha 147), same data; 2 우 1 juv. in MRAC (217296 part), Kakum Forest, secondary forest, beating, 23 Nov. 2005 (R. Jocqué, D. de Bakker, L. Baert); 1 ^ิ 1 q (2 vials) in MRAC $(217260,276)$, same data but beating

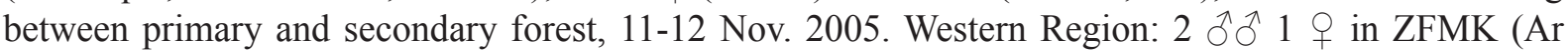
10496), Ankasa National Park $\left(5^{\circ} 13.0^{\prime} \mathrm{N}, 2^{\circ} 39.1^{\prime} \mathrm{W}\right), 180 \mathrm{~m}$ a.s.1., forest near entrance, night collecting, 22 Feb. 2013 (B.A. Huber); 2 우 in ZFMK (Ar 10497), Ankasa National Park, forest along Big Tree Trail ( $\left.\sim 5^{\circ} 15.1^{\prime} \mathrm{N}, 2^{\circ} 38.4^{\prime} \mathrm{W}\right), \sim 100 \mathrm{~m}$ a.s.1., 23 Feb. 2013 (B.A. Huber). Eastern Region: 1 q 2 juvs in ZFMK (Ar 10498), Atewa Hills, Atewa Atwirebu Reserve (6¹3.8’ N, $\left.0^{\circ} 33.5^{\prime} \mathrm{W}\right), 740$ m a.s.1., 25 Feb. 2013 (B.A. Huber); 1 i in pure ethanol, in ZFMK (Gha 135), same data.

21. Pholcus kindia Huber, 2011. Guinea (Huber 2011b).

Physocyclus Simon, 1893

Physocyclus is a New World genus. Only the pantropical synanthropic $P$. globosus (Taczanowski, 1874) occurs in West Africa (Fig. 31).

22. Physocyclus globosus (Taczanowski, 1874). Guinea, Ivory Coast (Millot 1941); newly recorded for Sierra Leone, Liberia, Ghana, Togo, and Benin.

\section{New records}

GUINEA: Guinée Forestière: 2 đ̊ 3 3 우 1 juv. in ZFMK (Ar 10499), Lola, in building $\left(7^{\circ} 48.5^{\text {' }} \mathrm{N}\right.$, $\left.8^{\circ} 30.9^{\prime} \mathrm{W}\right), 490$ m a.s.1., 30 Nov. 2008 (B.A. Huber).

SIERRA LEONE: 25 ठึ 35 우 (5 vials) in MRAC (146489, 148459, 148462, 148541, 159145), Freetown, Mount Aureol [8²8.7’N, 13ํ'13.3’W], Sep. 1976-Nov. 1977 (D. Olu-Pitt).

LIBERIA: Montserrado Co.: 4 `̊ $0^{\wedge} 6$ 우 + juvs (6 vials) in USNM, Monrovia ( $\left.6^{\circ} 19^{\prime} \mathrm{N}, 10^{\circ} 48^{\prime} \mathrm{W}\right)$, Jun.

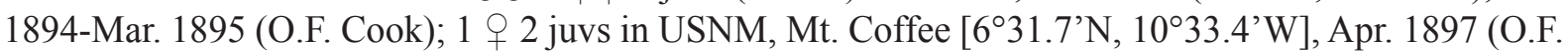
Cook). Maryland Co.: 2 우 + juvs in USNM, Muhlenberg Mission ( $\left.4^{\circ} 28^{\prime} \mathrm{N}, 7^{\circ} 35^{\prime} \mathrm{W}\right)$, Feb. 1895 (G.P. Goff); 1 ㅇ 1 juv. in USNM, same locality, Jun. 1892 (O.F. Cook); 1 ㅇ in USNM, unidentified locality: Liberia, "N.Y. settlement", Mar. 1895 (J.S. Sharp).

IVORY COAST: 1 i in MRAC (201102), Adiopo Doumé [5²0’ N, 4²0’W], Orstom, around buildings, 12 Nov. 1994 (R. Jocqué); 1 đo in MRAC (177559), Appouesso, Forêt classée de la Bossematié [6³5’N, 


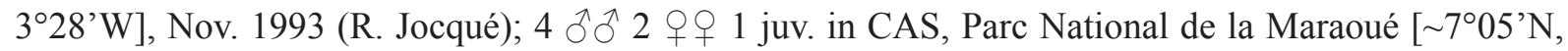
$\left.6^{\circ} 05^{\prime} \mathrm{W}\right], 8$ Jan. 1991 (W.J. Pulawski); 1 क in MRAC (230443), Taï Forest, Centre de Recherche Ecologique (CRE) [ $\left.5^{\circ} 50^{\prime} \mathrm{N}, 7^{\circ} 21^{\prime} \mathrm{W}\right]$, no further data.

GHANA: Greater Accra Region: 2 우 2 juvs in MRAC (131433), Accra [ $\left.\sim 5^{\circ} 35^{\prime} \mathrm{N}, 0^{\circ} 12^{\prime} \mathrm{W}\right], 2-8$ Oct. 1966 (D. Thys van den Audenaerde); 1 o in MRAC (127241), same locality, 20 Jul. 1964 (G. Marlier);

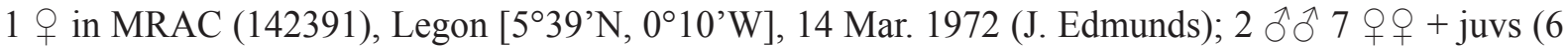
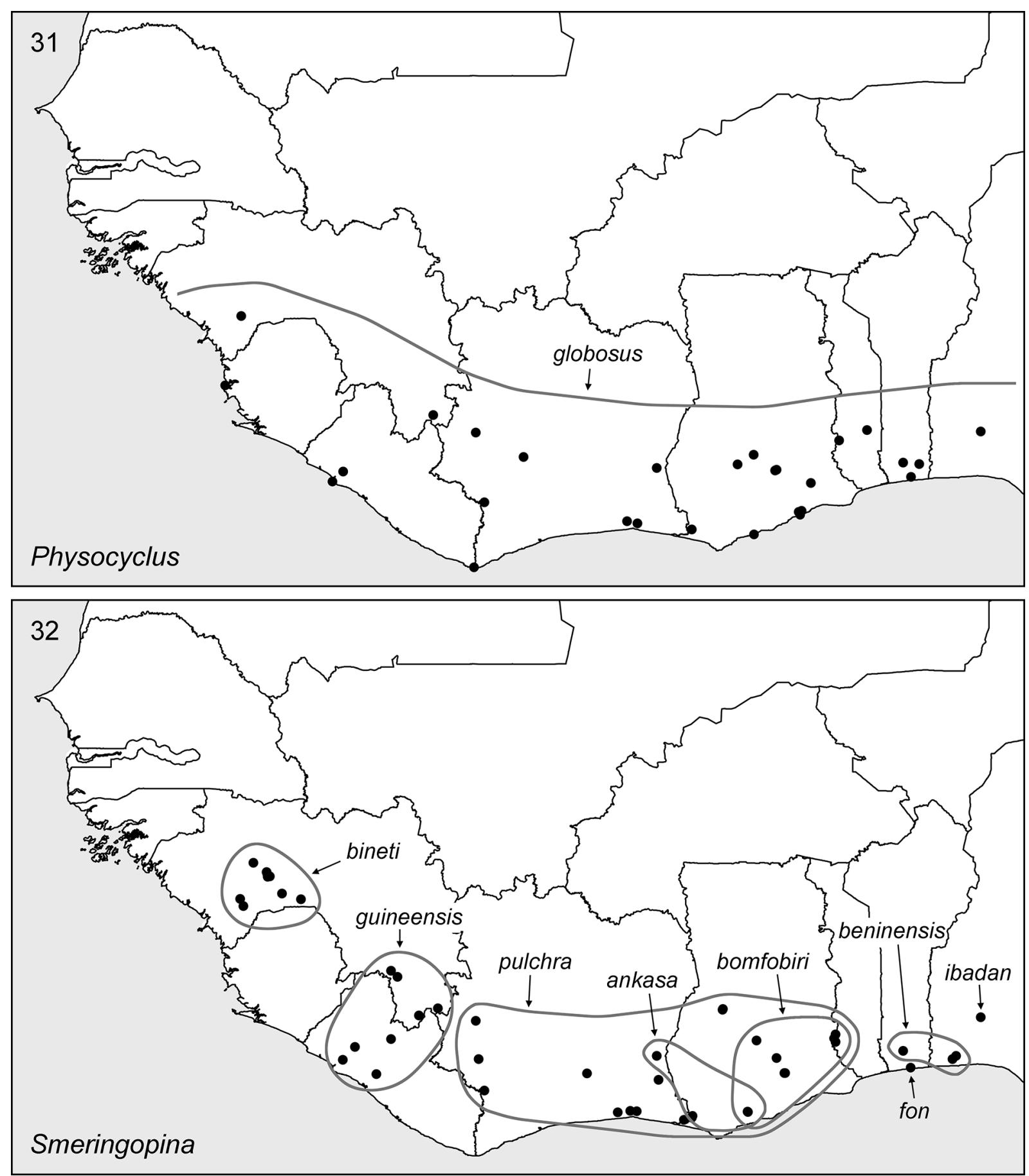

Figs 31-32. Known distributions of the genera Physocyclus Simon, 1893 and Smeringopina Kraus, 1957 in West Africa. 


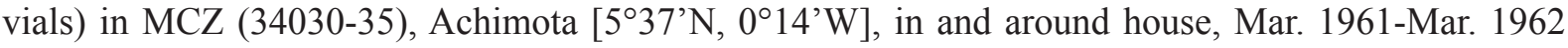

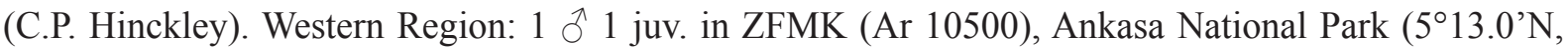
$2^{\circ} 39.1^{\prime} \mathrm{W}$ ), $180 \mathrm{~m}$ a.s.l., buildings near entrance, 22 Feb. 2013 (B.A. Huber). Central Region: 1 in ZFMK (Ar 10501), Cape Coast $\left(5^{\circ} 06.3^{\prime} \mathrm{N}, 1^{\circ} 14.8^{\prime} \mathrm{W}\right), 15 \mathrm{~m}$ a.s.1., in building, 23 Feb. 2013 (B.A. Huber). Eastern Region: 5 ภ $370 \mathrm{~m}$ a.s.l., degraded forest near road, at rocks, 10 Mar. 2013 (B.A. Huber); 3 우 1 juv. in pure ethanol, in ZFMK (Gha 151), same data; 1 o 1 juv. in CAS, Nkawkaw [6³3’N, $0^{\circ} 46^{\prime} \mathrm{W}$ ], 31 Jan. 1991 (W.J. Pulawski); $10^{\top}$ in MRAC (127253), Akosombo [6 $\left.6^{\circ} 16^{\prime} \mathrm{N}, 0^{\circ} 03^{\prime} \mathrm{E}\right], 12$ Jul. 1964 (G. Marlier). Ashanti Region: 4 ภำ 3 우 in ZFMK (Ar 10503), Kumawu ( $\left.6^{\circ} 54.3^{\prime} \mathrm{N}, 1^{\circ} 15.4^{\prime} \mathrm{W}\right), 380 \mathrm{~m}$ a.s.1., in building, 3 Mar. 2013 (B.A. Huber); 1 q in MRAC (131424), Kumasi [6²41’N, 1³7’W], 24-25 Sep. 1966 (D. Thys van den Audenaerde).

TOGO: several $\widehat{\partial}^{\lambda}{ }^{\Uparrow}$ 웅 (3 vials) in MRAC (136122-123, 150069), Kolokope [7²8’N, $\left.1^{\circ} 19^{\prime} \mathrm{E}\right], 26-31$ Jul. 1969 (F. Puylaert); 1 q 2 juvs in MRAC (131453), "Doteleopé", 6-7 Oct. 1966 (D. Thys van den Audenaerde); 1 ㅇ in MRAC (212772), Dzogbégan [7¹4’N, $0^{\circ} 41^{\prime} \mathrm{E}$ ], Jan. 2003 (S. Tchibozo).

BENIN: $1{ }^{\prime}$ in MRAC (212766 part), Adjohoun [6 $6^{\circ} 42^{\prime} \mathrm{N}, 2^{\circ} 30^{\prime} \mathrm{E}$ ], in house, 23 Dec. 2002 (S. Tchibozo); 2 우 1 juv. in MRAC (212769), Godomey [6 $\left.6^{\circ} 5^{\prime} \mathrm{N}, 2^{\circ} 19^{\prime} \mathrm{E}\right]$, in house, Dec. 2002 (S. Tchibozo); 2 우 in ZFMK (Ar 10504), Niaouli (644’N, 208'E), 20 Feb. 2009 (S. Tchibozo).

NIGERIA: Osun: 1 đ 1 + 1 juv. in MRAC (174602 part), Ile-Ife, Obafemi Awolowo Univ. campus [7²8’N, 434’E], 1991 (H. Segers).

\section{Quamtana Huber, 2003}

Quamtana is most diverse in southern Africa, but a few species occur as far north as Cameroon, Congo DR, Uganda (Huber 2003c), Rwanda ["Burundi" in Huber \& Warui 2012 is a lapsus; Cyamudongo is in Rwanda, $\left.2^{\circ} 33.5^{\prime} \mathrm{S}, 28^{\circ} 59.5^{\prime} \mathrm{E}\right]$ and Kenya (Huber \& Warui 2012). The new records below include the first for West Africa, and indicate that Q. kitahurira Huber, 2003 (previously known from Uganda and Rwanda only) is apparently a widespread species.

23. Quamtana kitahurira Huber, 2003. newly recorded for West Africa (Guinea) (see page 38).

\section{Smeringopina Kraus, 1957}

The Guineo-Congolian genus Smeringopina is highly diverse in Central Africa but only eight species are known from West Africa (Huber 2013; Fig. 32). Most species belong to one of the two endemic West African species groups, the guineensis group and the ankasa group (Huber 2013); only S. beninensis Kraus, 1957 is a notable outlier of a group otherwise restricted to Central Africa.

24. Smeringopina ankasa Huber, 2013. Ghana, Ivory Coast (Huber 2013).

25. Smeringopina beninensis Kraus, 1957. Benin, Nigeria (Kraus 1957; Huber 2013).

26. Smeringopina bineti (Millot, 1941). Guinea (Millot 1941; Huber 2013).

27. Smeringopina bomfobiri Huber, 2013. Ghana (Huber 2013).

28. Smeringopina fon Huber, 2013. Benin (Huber 2013).

29. Smeringopina guineensis (Millot, 1941). Guinea, Liberia (Millot 1941; Huber 2013).

30. Smeringopina ibadan Huber, 2013. Nigeria (Huber 2013). 
HUBER B.A. \& KWAPONG P., West African pholcid spiders

31. Smeringopina pulchra (Millot, 1941). Guinea(?), Ivory Coast, Ghana, Togo (Millot 1941; Huber 2013).

Smeringopus Simon, 1890

Smeringopus is currently the most species-rich African pholcid genus, but only two species occur in West Africa (Fig. 33): the pantropical synanthropic S. pallidus (Blackwall, 1858) and the widespread Guineo-Congolian S. cylindrogaster (Simon, 1907) which covers the entire Guineo-Congolian rainforest (Huber 2012).

32. Smeringopus cylindrogaster (Simon, 1907). Guinea Bissau, Guinea, Liberia, Ivory Coast, Ghana (Simon 1907; Huber 2009, 2012).

\section{New records}

GUINEA: 1 1 juv. in pure ethanol, in MRAC (236933), Mt. Nimba, Gouan Forest Centre [742'N, $\left.8^{\circ} 24^{\prime} \mathrm{W}\right]$, beating trees and shrubs with hanging litter at 1.5-3 $\mathrm{m}$ above ground, $1250 \mathrm{~m}$ a.s.1., 8 Oct. 2011 (D. Van den Spiegel, A. Henrard).

IVORY COAST: 1 q in MRAC (230346 part), Taï Forest, Centre de Recherche Ecologique (CRE) [5 $5^{\circ}{ }^{\prime} \mathrm{N}, 7^{\circ} 21^{\prime} \mathrm{W}$ ], beating in forest near Chimpanzee Camp, 22 Feb. 2010 (R. Jocqué, M. Diarassouba); 1 i in MRAC (230146 part), same locality, at foot of trees, by hand, 22 Feb. 2010 (R. Jocqué); 1 ㅇ 1 juv. in MRAC (230281), same data but forest near river, 22 Feb. 2010 (R. Jocqué, M. Diarassouba); 1 in MRAC (230244), same locality, forest E of camp, "layon Gérard", inundated forest, sieved litter, 20 Feb. 2010 (R. Jocqué, L. Oulaii); 2 q 9 in MRAC (230296), same locality, forest across river, forest on clayey soil, beating, 20 Feb. 2010 (R. Jocqué, M. Diarassouba); 1 in MRAC (233643), Taï Forest, beating, 1 Sep. 2010 (D. Van den Spiegel, A. Kablan); 2 juvs in MRAC (230360 part), Adiopo Doumé $\left[5^{\circ} 20^{\prime} \mathrm{N}, 4^{\circ} 20^{\prime} \mathrm{W}\right]$, Centre Suisse de Recherche Scientifique (CSRS), small dry forest, sieving litter, 26 Feb. 2010 (D. van den Spiegel, R. Jocqué).

GHANA: Central Region: 1 in pure ethanol, in ZFMK (Gha 149), Kakum National Park (5⒛9'N, $\left.1^{\circ} 23.0^{\prime} \mathrm{W}\right), 160 \mathrm{~m}$ a.s.l., forest near entrance, day collecting, 19 Feb. 2013 (B.A. Huber); 1 ऽ 1 ㅇ 1 juv. in MRAC (217296 part), Kakum Forest, beating in secondary forest, 23 Nov. 2005 (R. Jocqué, D. de Bakker, L. Baert). Eastern Region: 3 우 in ZFMK (Ar 10505), Atewa Hills, Atewa Atwirebu Reserve $\left(6^{\circ} 13.8^{\prime} \mathrm{N}, 0^{\circ} 33.5^{\prime} \mathrm{W}\right), 740 \mathrm{~m}$ a.s.1., 25 Feb. 2013 (B.A. Huber); 1 ○ in ZFMK (Ar 10506), near Mpraeso $\left(6^{\circ} 34.1^{\prime} \mathrm{N}, 0^{\circ} 43.9^{\prime} \mathrm{W}\right), 370 \mathrm{~m}$ a.s.1., degraded forest near road, 10 Mar. 2013 (B.A. Huber). Western Region: $1 \mathrm{O}^{\top}$ in ZFMK (Ar 10507), Ankasa National Park $\left(5^{\circ} 13.0^{\prime} \mathrm{N}, 2^{\circ} 39.1^{\prime} \mathrm{W}\right), 180 \mathrm{~m}$ a.s.1., forest near entrance, day collecting, 22 Feb. 2013 (B.A. Huber); 3 소 5 우 1 juv. (2 vials) in ZFMK (Ar 1050809), same data but night collecting; 1 ठ 1 juv. in pure ethanol, in ZFMK (Gha 157), same data.

33. Smeringopus pallidus (Blackwall, 1858). Guinea, Ivory Coast (Millot 1941); newly recorded for Senegal, The Gambia, Sierra Leone, and Ghana.

\section{New records}

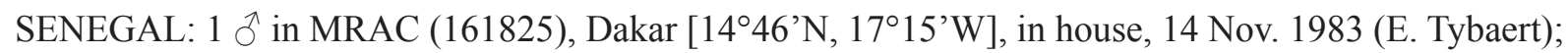

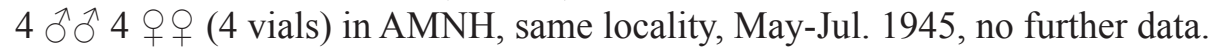

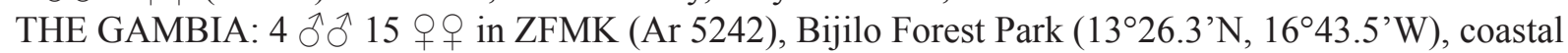
forest, 22 Oct. 2008 (Yu.M. Marusik); $10^{\top} 5$ + 9 in ZFMK (Ar 5244), same data, litter, mostly under

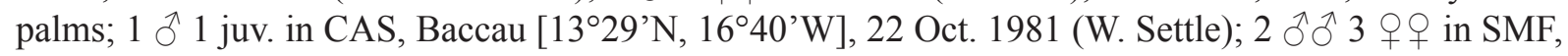
same locality, in building, no date (J. Wunderlich).

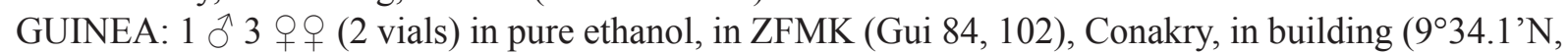
13³9.7’W), 40 m a.s.1., 18 Nov. 2008 (B.A. Huber). Moyenne-Guinée: 1 o in ZFMK (Ar 10510), Dalaba $\left(10^{\circ} 41.4^{\prime} \mathrm{N}, 12^{\circ} 15.7^{\prime} \mathrm{W}\right)$, in building, $1260 \mathrm{~m}$ a.s.1., 22 Nov. 2008 (B.A. Huber). MoyenneGuinée: 2 소 6 우우 in pure ethanol, in ZFMK (Gui 85), Doucki, in and at buildings $\left(10^{\circ} 59.1^{\prime} \mathrm{N}\right.$, 
12³6.0’W), 1050 m a.s.1., 23 Nov. 2008 (B.A. Huber). Basse-Guinée: 1 ð 1 juv. in ZFMK (Ar 10511), Kindia, in building $\left(10^{\circ} 03^{\prime} \mathrm{N}, 12^{\circ} 51^{\prime} \mathrm{W}\right), 400 \mathrm{~m}$ a.s.1., 20 Nov. 2008 (B.A. Huber).

SIERRA LEONE: 10 ๙ $\widehat{\jmath} 6$ 우 + juvs (3 vials) in MRAC (148460 part, 159113, 159145 part), Freetown,

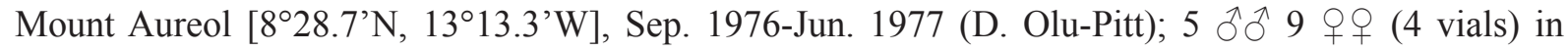
MRAC (146506, 148525, 530, 542), Freetown, Dec. 1974-Jan. 1977 (D. Olu-Pitt).
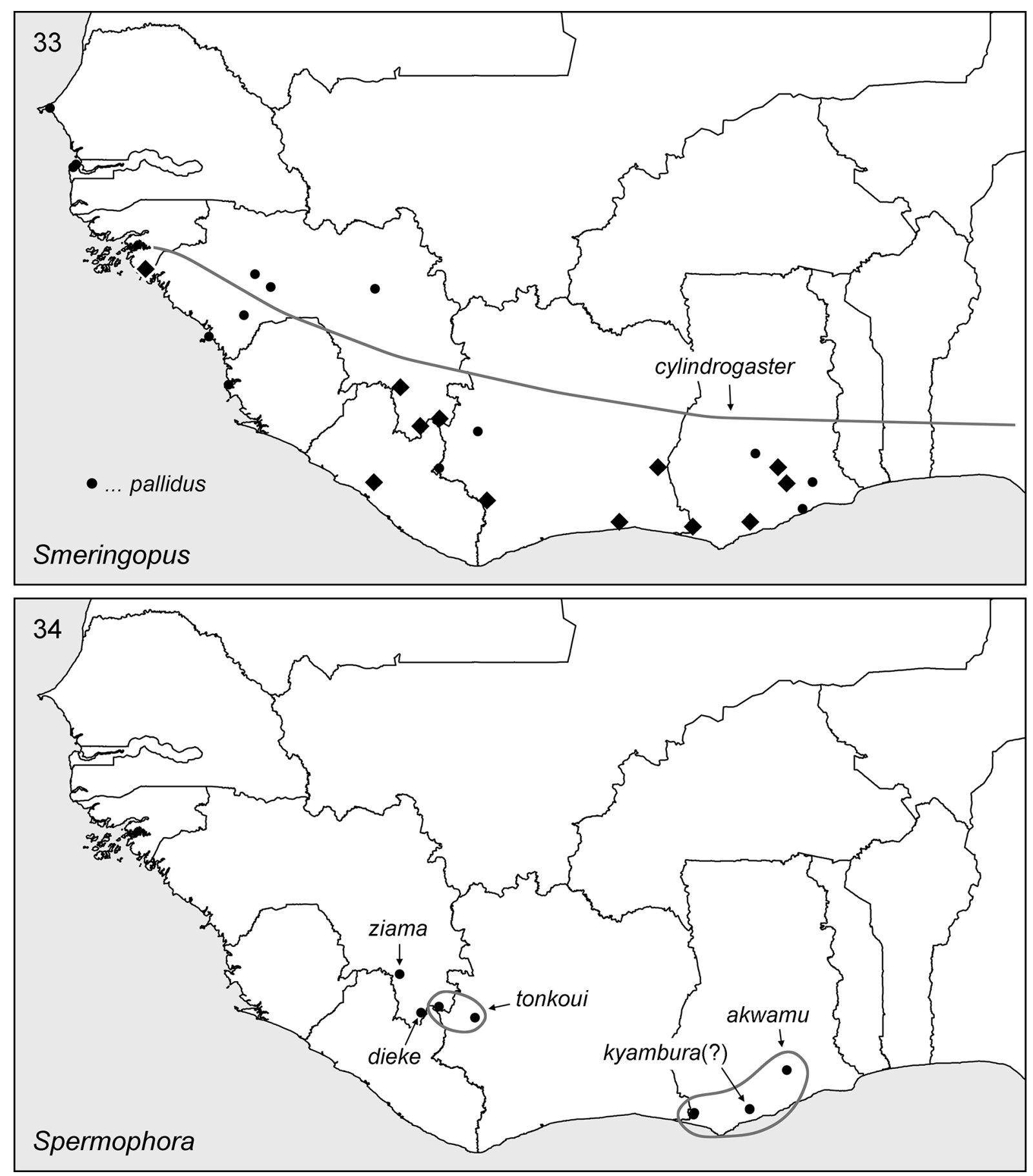

Figs 33-34. Known distributions of the genera Smeringopus Simon, 1890 and Spermophora Hentz, 1841 in West Africa. 


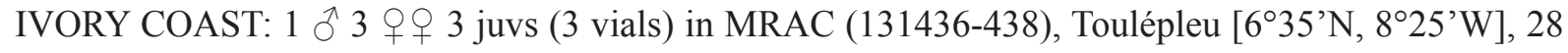
Jul. 1966 (W. Verheyen, D. Thys van den Audenaerde).

GHANA: Ashanti Region: 1 juv. in ZFMK (Ar 10512), Kumawu (6 $\left.6^{\circ} 54.3^{\prime} \mathrm{N}, 1^{\circ} 15.4^{\prime} \mathrm{W}\right), 380 \mathrm{~m}$ a.s.1., in building, 3 Mar. 2013 (B.A. Huber). Greater Accra Region: 1 ô in MRAC (142403), Legon [5³9'N, $0^{\circ} 10^{\prime} \mathrm{W}$ ], 14 Mar. 1972 (J. Edmunds). Eastern Region: 1 q in MRAC (127252), Akosombo [6ำ'N, 003’E], 12 Jul. 1964 (G. Marlier).

Spermophora Hentz, 1841

Spermophora is diverse in sub-Saharan Africa but relationships among African taxa and between African and Asian taxa remain unclear (Huber 2003b, 2003c, 2005; Dimitrov et al. 2013). Five species are known from West Africa (Fig. 34). Of these, S. tonkoui Huber, 2003 and S. ziama sp. nov. share several derived characters but their relationship to other conspecifics remain dubious (see under S. ziama sp. nov. description below). Another pair of putatively close relatives is $S$. dieke Huber, 2009 and $S$. akwamu sp. nov. (see under $S$. akwamu sp. nov. description below). A very similar undescribed species occurs in Congo DR (in MRAC). Finally, S. kyambura is a widespread Guineo-Congolian species but West African specimens were assigned tentatively (Huber \& Warui 2012).

34. Spermophora akwamu sp. nov. (see page 31). Ghana.

35. Spermophora dieke Huber, 2009. Guinea (Huber 2009).

36. Spermophora kyambura Huber \& Warui, 2012. Ghana (Huber \& Warui 2012).

37. Spermophora tonkoui Huber, 2003. Ivory Coast (Huber 2003b). Newly recorded for Guinea.

\section{New record}

GUINEA: 2 đิ $\widehat{0} 5$ 우 in pure ethanol, in MRAC (236928), Mt. Nimba, Forêt de Zié, near Gouan camp or "Station de Pompage Zié" [ $7^{\circ} 40^{\prime} \mathrm{N}, 8^{\circ} 26^{\prime} \mathrm{W}$ ], $1250 \mathrm{~m}$ a.s.l., beating trees and shrubs with hanging litter, at 1.5-3m above ground, 11 Oct. 2011 (D. Van den Spiegel, A. Henrard); 2 đ̊ 0 6 + 2 juvs (3 vials) in SMF, Mt. Nimba, Crête de Nion, 1100-1160 m a.s.1., 11 Feb.-12 May 1957 (M. Lamotte).

38. Spermophora ziama sp. nov. (see page 34). Guinea.

\section{Identification key to West African pholcid genera}

References to figures in upper case ("Fig.") refer to illustrations herein; figures in lower case ("fig.") have been published previously and are freely accessible online (at http://www.uni-bonn.de/ bhuber1/).

1. Abdomen worm-shaped ( $>10 \times$ longer than wide; Figs 4-5); male chelicerae with one pair of small projections in latero-distal position (Fig. 74) Leptopholcus Simon, 1893

- Abdomen not worm-shaped $(<10 \times$ as long as wide); male chelicerae different 2

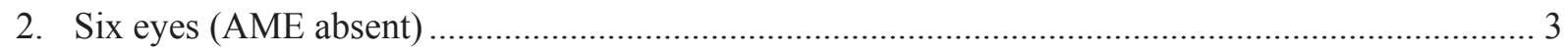

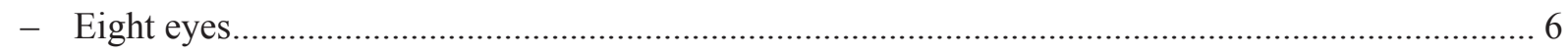

3. Sternum with characteristic pattern of radiating lines (Fig. 40); abdomen dorsally with dark heartmark (Fig. 35) Anansus Huber, 2007

- Sternum without pattern; abdomen dorsal pattern different. 
4. Carapace with 2-3 pairs of dark lateral spots; ocular area (especially in males) strongly elevated, in male with frontal hairy pocket; male chelicerae without proximal lateral projections (figs 2-4 in Huber 1996) Modisimus culicinus (Simon, 1893)

- Carapace without lateral spots; ocular area not elevated; male chelicerae with proximal lateral projections.

5. Procursus with highly complex system of transparent lamellae (fig. 14 in Huber 2007); male chelicerae narrowing distally, with pair of simple frontal apophyses close to median line (fig. 15 in Huber 2007)

.Nyikoa limbe Huber, 2007

- Procursus and male chelicerae different Spermophora Hentz, 1841

6. Carapace with median indentation (furrow or pit) 7

- Carapace evenly domed, without median indentation

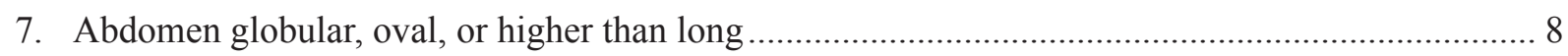

- Abdomen elongated. 9

8. Procursus with prominent distal spine; female carapace with posterior median cone acting against frontal plate on abdomen; epigynum with median anterior process.

Physocyclus globosus (Taczanowski, 1874)

- Procursus short, without distal spine (Figs 57-58); female carapace without posterior cone; epigynum without median anterior process (Fig. 54)...... Artema Walckenaer, 1837

9. Legs with many small black marks; abdomen angular in lateral view (posteriorly high; Fig. 16) ..... Crossopriza Simon, 1893

- Legs without small black marks, abdomen not angular in lateral view (posteriorly tapering)........ 10

10. Male chelicerae with proximal lateral projections, either without or with several modified hairs on each side.

Smeringopina Kraus, 1957

- Male chelicerae without proximal lateral projections, with single modified hair on each distal apophysis (fig. 47 in Huber 2009)

Smeringopus Simon, 1890

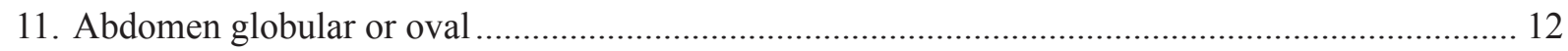

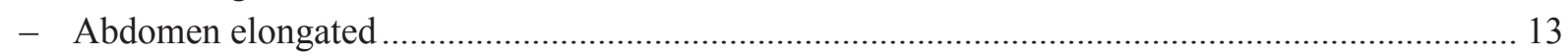

12. Procursus with long hinged dorsal process, epigynum weakly sclerotized, internal U-shaped structure visible through cuticle anteriorly (figs 83-89 in Huber 2011b)

Micropholcus fauroti (Simon, 1887)

- Procursus widely curved, without long dorsal process (figs 204-205 in Huber 2003c); epigynum weakly sclerotized, no internal structure visible through cuticle (Fig. 107)

Quamtana kitahurira Huber, 2003

13. Abdomen drawn into cone dorso-posteriorly (Fig. 8; fig. 29 in Huber 2011b); male chelicerae with pair of lateral unsclerotized projections in distal position (fig. 130 in Huber 2009); epigynum weakly sclerotized ................................ Pehrforsskalia conopyga Deeleman-Reinhold \& van Harten, 2001

- Abdomen rounded posteriorly; male chelicerae with pair of lateral unsclerotized projections in proximal position; epigynum strongly sclerotized

..Pholcus Walckenaer, 1805 


\title{
Taxonomy
}

\author{
Anansus atewa sp. nov. \\ urn:1sid:zoobank.org:act:9EC8FF7E-F56B-4FAD-9B48-730CE2795474
}

Figs 9-10, 23, 35-47

\section{Diagnosis}

Easily distinguished from known congeners by distinct pair of projections on male clypeus (Figs 35, 39; other known species with single tiny median process); also by male cheliceral apophyses (more proximal, wider apart, and more pointed than in other known species; Figs 39, 45), by shape of procursus (large ventral curved sclerite; without retrolateral sclerite provided with brush of long hairs; without ventral pointed projection; Figs 38, 44), and by more anterior position of epigynal pockets (Figs 41, 46).

\section{Etymology}

The species name is a noun in apposition, derived from the type locality.

\section{Type material}

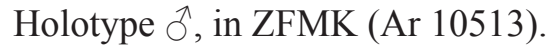

\section{Type data}

GHANA: Eastern Region: Atewa Hills, Atewa Atwirebu Reserve (6 $\left.6^{\circ} 13.8^{\prime} \mathrm{N}, 0^{\circ} 33.5^{\prime} \mathrm{W}\right), 740 \mathrm{~m}$ a.s.l., 25 Feb. 2013 (B.A. Huber).

\section{Other material examined}

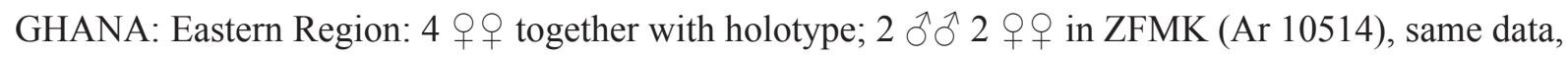
specimens taken alive and died from heat; 2 우 5 juvs in pure ethanol in ZFMK (Gha 137), same data.

\section{Description}

\section{Male (holotype)}

Measurements. Total body length 1.5, carapace width 0.6. Leg 1: $5.2(1.3+0.2+1.4+1.6+0.7)$, tibia 2: 0.9, tibia 3: 0.7, tibia 4: 1.1; tibia $1 \mathrm{~L} / \mathrm{d}: 22$. Distance PME-PME $25 \mu \mathrm{m}$, diameter PME $80 \mu \mathrm{m}$, distance PME-ALE $20 \mu \mathrm{m}$, no AME.

COLOR. Carapace ochre-grey, slightly darker medially, sternum with four pairs of dark lines extending from behind labium to bases of leg coxae, legs pale ochre-yellow, without rings, abdomen ochre-gray with distinct dark median dorsal mark.

Body. Habitus as in Figs 35-36; ocular area barely elevated; carapace without median furrow; clypeus with distinct pair of projections at rim (Fig. 35); sternum wider than long (0.40/0.35), unmodified. Chelicerae as in Fig. 45, with pair of lateral processes proximally and pointed frontal apophyses, without stridulatory ridges.

PALPS. As in Figs 37-38 and 43-44, coxa unmodified, trochanter with indistinct ventral projection, femur small relative to tibia, with small retrolatero-ventral process and larger prolatero-ventral process set with tubercles, procursus complex, entire distal part apparently hinged against basis, without elements distinctive for other known species (retrolateral sclerite provided with brush of long hairs; ventral pointed projection), bulb with single weakly sclerotized process (embolus). 
LEGs. Without spines and curved hairs, few vertical hairs; retrolateral trichobothrium on tibia 1 at $25 \%$; prolateral trichobothrium absent on tibia 1, present on other tibiae; tarsus 1 with about ten indistinct pseudosegments.

VARIATION. Tibia 1 in 2 other males: $1.3,1.4$.

\section{Female}

In general similar to male; tibia 1 in 6 females: 1.2-1.4 (mean 1.3). Epigynum consisting of large anterior plate provided with pair of pockets and narrow posterior plate (Figs 40, 41, 46); internal genitalia as in Figs 42 and 47.

\section{Natural history}

The spiders were found in the leaf-litter under leaves that are curved in a way to produce protected space on the underside. Two eggsacs contained 13 and 15 eggs respectively.
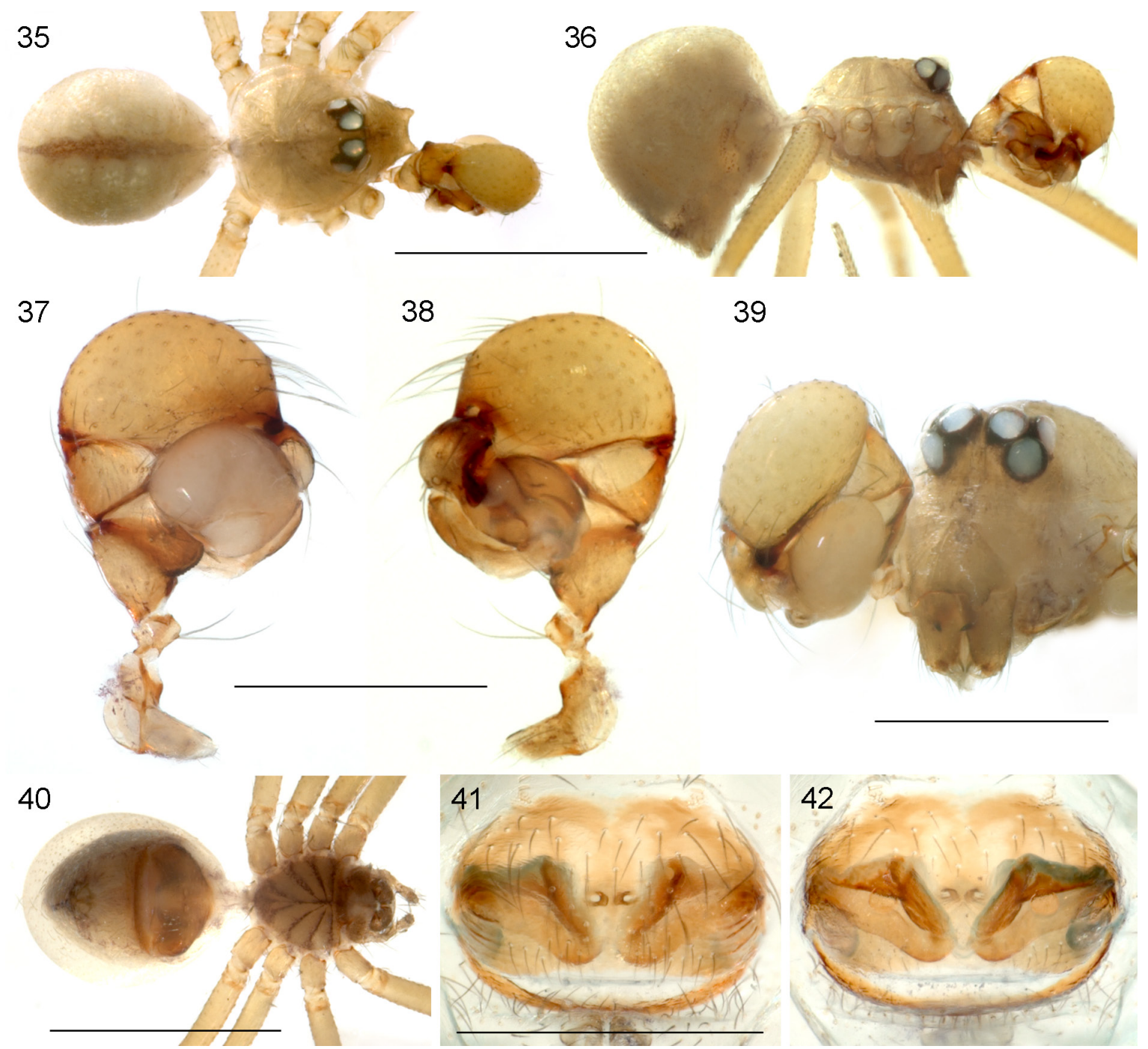

Figs 35-42. Anansus atewa sp. nov. 35-36. $\widehat{\jmath}$, dorsal and lateral views. 37-38. Left $\widehat{\jmath}$ palp, prolateral and retrolateral views. 39. Ô prosoma and right palp, oblique frontal view. 40. + , ventral view. 4142. Cleared q genitalia, ventral and dorsal views. Scale lines: $35-36,40=1 \mathrm{~mm} ; 37-39,41-42=$ $0.5 \mathrm{~mm}$. 


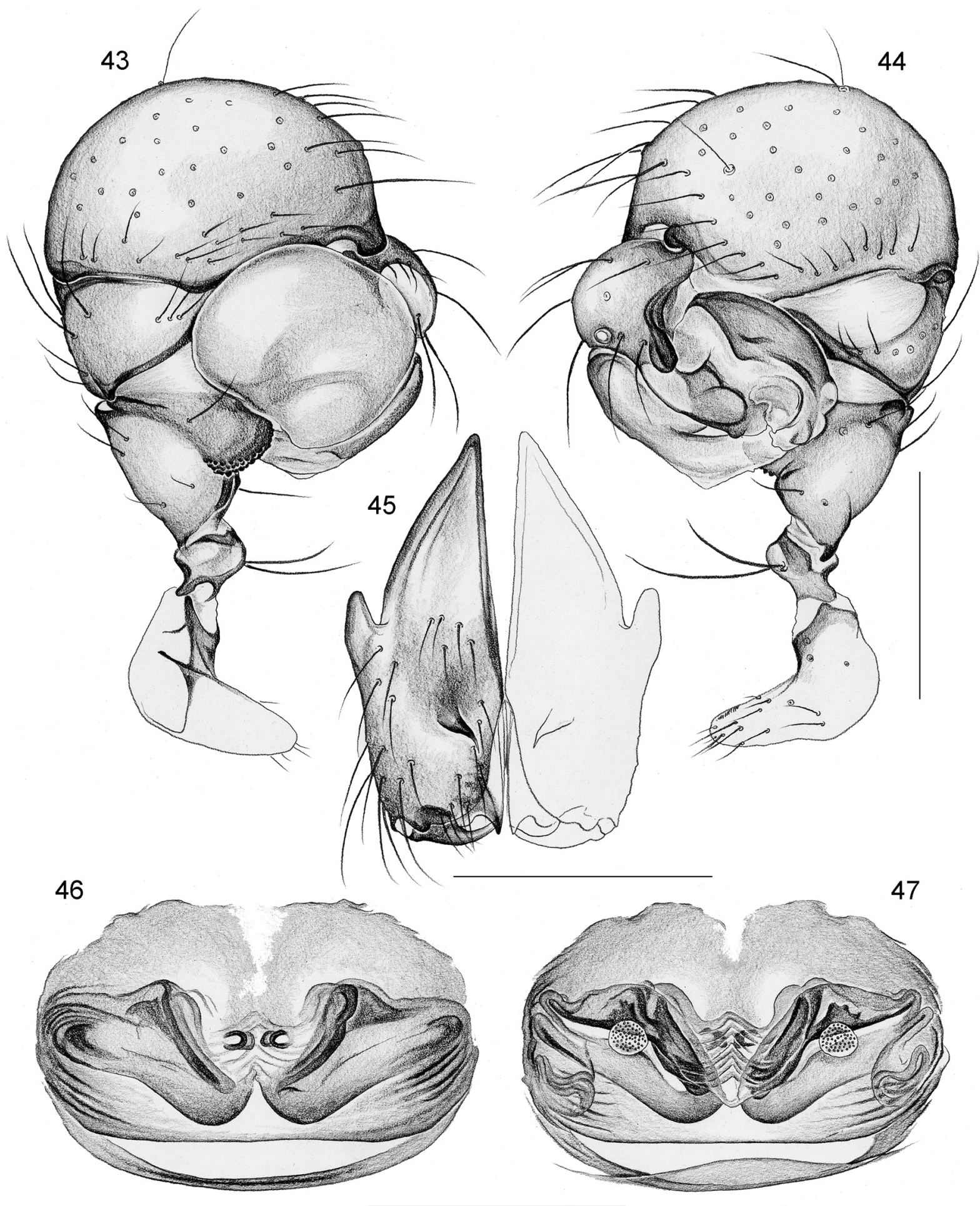

Figs 43-47. Anansus atewa sp. nov. 43-44. Left $ð$ palp, prolateral and retrolateral views. 45 . $\widehat{\partial}$ chelicerae, frontal view. 46-47. Cleared + genitalia, ventral and dorsal views. Scale lines: 43-44, 46-47 =0.3 mm; $45=0.2 \mathrm{~mm}$. 


\section{Distribution}

Known from type locality only (Fig. 23). Further juvenile specimens from near Mpraeso and from Tagbo Falls (Ghana; in ZFMK) might also belong to this species.

\section{Artema bunkpurugu sp. nov. urn:1sid:zoobank.org:act:05986623-C648-495E-AA88-C708FEF1A926}

Figs 14-15, 24, 48-62

"Artema mauriciana" (misidentification) - Millot 1941: 3-5, figs 1A-E, G-I (fig. 1F is the true $A$. mauriciana $=$ A. atlanta).

\section{Diagnosis}

Distinguished from the widespread A. atlanta by shape of procursus (arrows in Figs 57-58), bulbal processes (arrow in Fig. 58), relatively longer male palpal tibia, less prominent male cheliceral projections, more straight posterior epigynal margin (Figs 54, 61), and more prominent anterior epigynal projections (Fig. 49). Middle East and Central Asian species have a very different epigynum (cf. fig. 10 in Spassky 1934).

\section{Etymology}

The species name is a noun in apposition, derived from the type locality.

\section{Type material}

Holotype ${ }^{\Uparrow}$, in ZFMK (Ar 10515).

\section{Type data}

GHANA: Northern Region: Bunkpurugu ( $\left.10^{\circ} 31.1^{\prime} \mathrm{N}, 0^{\circ} 05.5^{\prime} \mathrm{E}\right), 230 \mathrm{~m}$ a.s.1., in building, 7 Mar. 2013 (B.A. Huber).

\section{Other material examined}

GHANA: Northern Region: $1 q$ together with holotype; 2 qq 1 juv. in pure ethanol in ZFMK (Gha 126), same data; 1 ㅈ 2 우우 in ZFMK (Ar 10516), between Bunkpurugu and Nakpanduri (10³2.9' $\mathrm{N}$, $\left.0^{\circ} 00.2^{\prime} \mathrm{E}\right), 270 \mathrm{~m}$ a.s.1., under concrete bridge, 8 Mar. 2013 (B.A. Huber); 1 q in pure ethanol in ZFMK (Gha 128), same data; 1 \% in ZFMK (Ar 10517), near Bimbaga $\left(10^{\circ} 34.5^{\circ} \mathrm{N}, 0^{\circ} 03.8^{\prime} \mathrm{W}\right), 330 \mathrm{~m}$ a.s.l., under concrete bridge, 8 Mar. 2013 (B.A. Huber); 2 우 in ZFMK (Ar 10518), near Nakpanduri $\left(10^{\circ} 35.0^{\prime} \mathrm{N}, 0^{\circ} 06.4^{\prime} \mathrm{W}\right), 345 \mathrm{~m}$ a.s.l., under concrete bridge, 8 Mar. 2013 (B.A. Huber); 1 q 2 juvs in pure ethanol in ZFMK (Gha 140), between Gambaga and Nakpanduri $\left(10^{\circ} 34.0^{\prime} \mathrm{N}, 0^{\circ} 17.5^{\prime} \mathrm{W}\right), 400 \mathrm{~m}$ a.s.l., under large rocks, 8 Mar. 2013 (B.A. Huber).

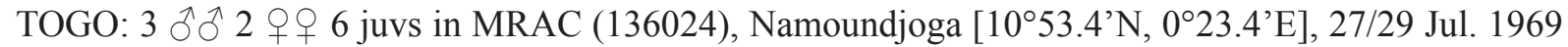
(F. Puylaert).

BURKINA FASO: Sud-Ouest: $1 \delta^{\wedge}$ in MRAC (128076), Ouagadougou [12²2’ N, $1^{\circ} 32^{\prime} \mathrm{W}$ ], Apr.-May

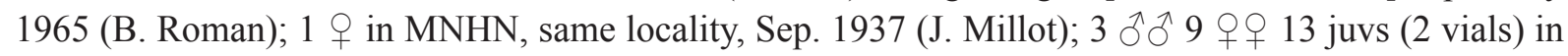
MNHN, Batié [952’N, 255’W], Sep. 1937 (J. Millot).

MALI: 1 q 3 juvs (?, abdomens missing in two specimens) in MNHN, Bamako [12 $38^{\prime} \mathrm{N}, 8^{\circ} 00^{\prime} \mathrm{W}$ ], Oct. 1937 (J. Millot); 1 ㅇ in AMNH, Gao [16¹6’N, 003’W], 23-25 Nov. 1948 (B. Malkin).

GUINEA: 1 ` (1 palp and chelicerae missing), 2 q + (1 epigynum missing), 2 juvs in MNHN, Kouroussa [10³9'N, 953'W], Oct. 1937 (J. Millot). 


\section{Description}

\section{Male (holotype)}

MEASUREMENTS. Total body length 9.0, carapace width 4.2. Leg 1: $77.1(20.3+1.9+21.1+29.1+4.7)$, tibia 2: 16.3, tibia 3: 12.7, tibia 4: 16.1; tibia $1 \mathrm{~L} / \mathrm{d}$ : 54. Distance PME-PME $230 \mu \mathrm{m}$, diameter PME $220 \mu \mathrm{m}$, distance PME-ALE $115 \mu \mathrm{m}$, distance AME-AME $60 \mu \mathrm{m}$, diameter AME $210 \mu \mathrm{m}$.
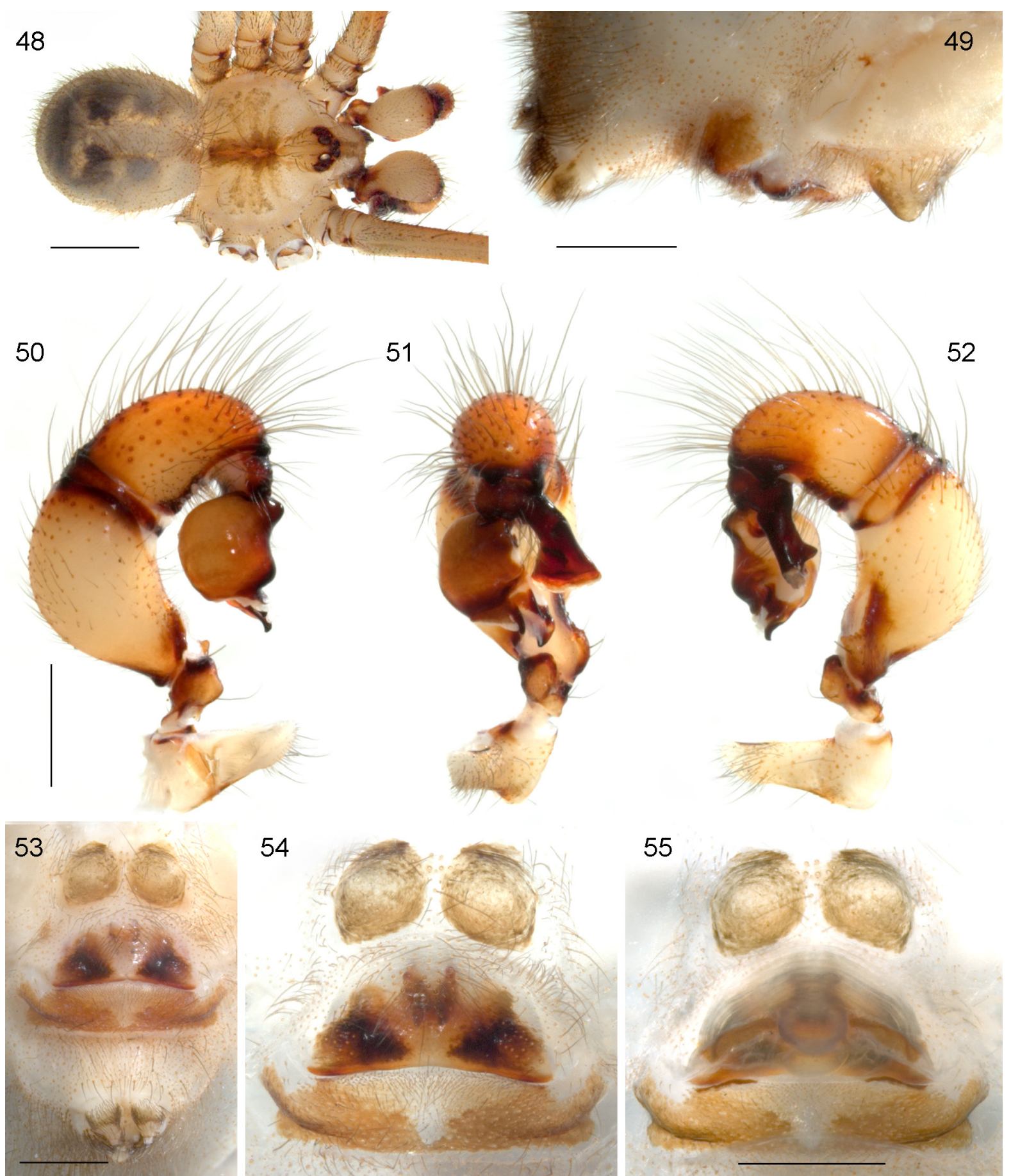

Figs 48-55. Artema bunkpurugu sp. nov. 48. $\widehat{\jmath}$, dorsal view. 49. Female genitalia and spinnerets, lateral view. 50-52. Left ${ }^{\lambda}$ palp, prolateral, dorsal, and retrolateral views. 53. + abdomen, ventral view. 5455. Cleared $q$ genitalia, ventral and dorsal views. Scale lines: $48=2 \mathrm{~mm} ; 49-55=1 \mathrm{~mm}$. 
CoLOR. Carapace pale ochre-grey with light brown median band and small brown spots laterally, clypeus with pair of narrow light brown bands below AME, legs pale ochre-gray to light brown, with dark rings on femora subdistally, patellae + tibiae proximally, and tibiae subdistally, tips of femora and tibiae whitish, sternum pale gray with narrow dark brown margins, abdomen pale gray with large dark marks dorsally.

Body. Habitus as in Fig. 48; ocular area slightly elevated; carapace with large median pit and distinct median furrow posteriorly; clypeus unmodified; sternum wider than long (2.5/2.0), unmodified. Chelicerae as in Figs 59-60, with heavily sclerotized frontal projections provided with about ten modified (cone-shaped) hairs each, without stridulatory ridges. Gonopore with five epiandrous spigots.

PALPS. As in Figs 50-52 and 56-58, coxa unmodified, trochanter with short ventral projection, femur with distinct retrolateral process proximally, large ventral membranous area proximally bordered on both sides by heavily sclerotized ridges, and small dorsal projection proximally; femur-patella hinges close together dorsally; patella very short; procursus with proximal dorsal apophysis and weakly developed ventral pocket, with distinctive ventral projection distally (arrow in Fig. 57), bulb with two apophyses flanking membranous process (putative embolus).

LEGs. Without spines, with some very weakly curved hairs on tibiae and metatarsi, few vertical hairs mainly on distal leg segments; retrolateral trichobothrium on tibia 1 at 5.5\%; prolateral trichobothrium present on all tibiae (also tibia 1); pseudosegmentation not visible.

VARIATION. Tibia 1 in 8 other males: 15.5-23.1 (mean 19.8). Lateral spots on carapace sometimes fused to three pairs of large marks. Sternum posteriorly with variably distinct pattern of light brown marks. Gonopore with variable number of epiandrous spigots (4-6).

\section{Female}

In general similar to male but chelicerae with distinct sclerotized patches laterally provided with stidulatory files (cf. fig. $1 \mathrm{G}$ in Millot 1941); tibia 1 in 20 females: 12.0-18.9 (mean 16.1). Epigynum consisting of trapezoidal anterior plate and simple posterior plate, with pair of large distinctive projections in front of epigynum (Figs 49, 53-54, 61); internal genitalia as in Figs 55, 62.

\section{Natural history}

The spiders were found both in natural habitats (among large rocks) and in human-made habitats (under low concrete bridges, in buildings; see also Millot 1941). When disturbed, the spiders try to hide in crevices, running short distances and then vibrating at low amplitude but high frequency.

\section{Distribution}

Widely distributed in Sudanian West Africa (Fig. 24).

\section{Note}

Millot (1941) was aware of the fact that most of his specimens from West Africa did not agree well with published figures of $A$. mauriciana $(=A$. atlanta). He tentatively interpreted this as intraspecific variation. However, comparison of numerous specimens of this new species with specimens of $A$. atlanta from all over the World shows that the two species differ in several characters (see above) and that no intermediate specimens seem to occur.

Millot's (1941) specimens from Kindia, Guinea (2 juvs; $q$ missing), Ferkessedougou, Ivory Coast ( 1 juv.), and Gao, Mali ( 2 juvs) are in MNHN but juveniles of $A$. atlanta and $A$. bunkpurugu sp. nov. are indistinguishable so their identity is unclear. 


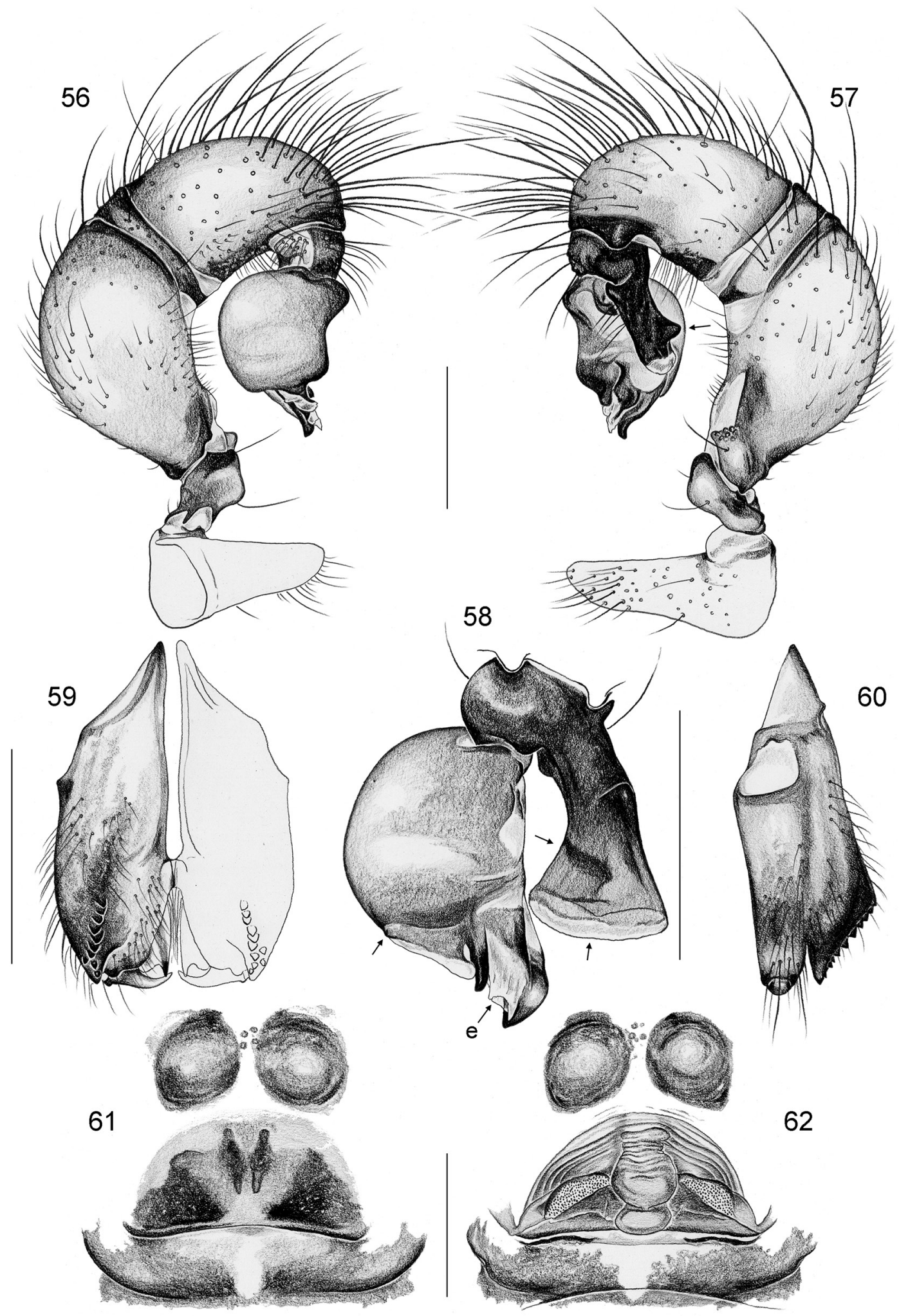

Figs 56-62. Artema bunkpurugu sp. nov. 56-57. Left $\widehat{o}$ palp, prolateral and retrolateral views. 58. Left procursus and genital bulb, dorsal view (e: embolus). 59-60. $\sigma^{\lambda}$ chelicerae, frontal and lateral views. 6162. Cleared $q$ genitalia, ventral and dorsal views. Arrows point at distinctive structures (as compared with $A$. atlanta). Scale lines: $1 \mathrm{~mm}$. 


\section{Leptopholcus kintampo sp. nov.}

urn:1sid:zoobank.org:act:AB6C8FC0-A0FB-4EFA-82A5-A622435F19CF

Figs 4, 26, 63-76

\section{Diagnosis}

Distinguished from similar close relatives (species with median process on male clypeus: L. signifer Simon, 1893; L. debakkeri Huber, 2011; L. guineensis; L. dschang Huber 2011; L. gracilis Berland, 1920; L. budongo Huber, 2011) by shapes of bulbal processes (Figs 68, 72).

\section{Etymology}

The species name is a noun in apposition, derived from the type locality.

\section{Type material}

Holotype ổ, in ZFMK (Ar 10519).

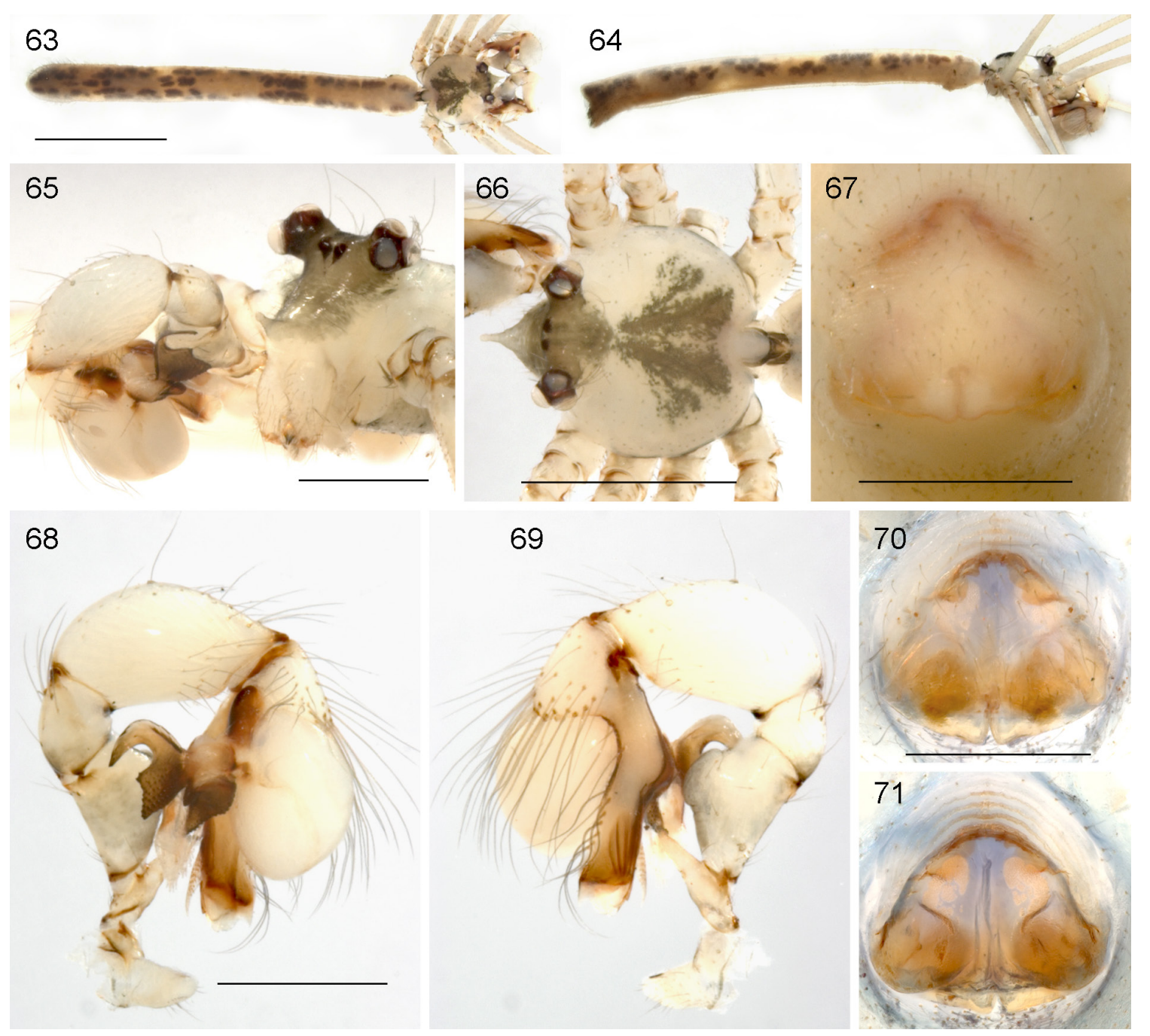

Figs 63-71. Leptopholcus kintampo sp. nov. 63-64. $\widehat{\partial}$, dorsal and lateral views. 65-66. $\widehat{\partial}$ prosoma, oblique frontal and dorsal views. 67. Epigynum, ventral view. 68-69. Left $\delta$ palp, prolateral and retrolateral views. 70-71. Cleared $q$ genitalia, ventral and dorsal views. Scale lines: 63-64 $=2 \mathrm{~mm}$; $66=1 \mathrm{~mm} ; 65,67-71=0.5 \mathrm{~mm}$. 


\section{Type data}

GHANA: Brong-Ahafo Region: Kintampo Falls $\left(8^{\circ} 05.3^{\prime} \mathrm{N}, 1^{\circ} 41.9^{\prime} \mathrm{W}\right), 280 \mathrm{~m}$ a.s.l., degraded forest along stream, 5 Mar. 2013 (B.A. Huber).

\section{Other material examined}

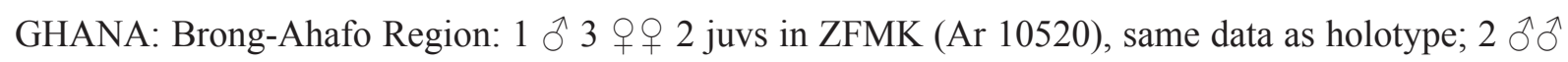
2 우 2 juvs in ZFMK (Ar 10521), same data but 9 Mar. 2013; 2 우 2 juvs in pure ethanol in ZFMK (Gha 161), same data as holotype.

\section{Description}

\section{Male (holotype)}

Measurements. Total body length 7.5, carapace width 1.1. Leg 1: $33.0(8.3+0.5+7.9+13.0+3.3)$, tibia 2: 5.9, tibia 3: 3.8, tibia 4: 6.0; tibia $1 \mathrm{~L} / \mathrm{d}: 89$. Distance PME-PME $345 \mu \mathrm{m}$, diameter PME $95 \mu \mathrm{m}$, distance PME-ALE $25 \mu \mathrm{m}$, diameter AME $25 \mu \mathrm{m}$, distance AME-AME $25 \mu \mathrm{m}$.

CoLoR. Carapace pale ochre-yellow with large triangular brown mark (Fig. 66), ocular area and clypeus also dark, sternum pale gray with dark margins and small dark spots, legs pale ochre-yellow with dark rings in patella area and at tibia-metatarsus joints, abdomen ochre-yellow with numerous black dorsal marks.

Body. Habitus as in Figs 63-64; ocular area slightly elevated, each triad on short stalk directed laterally (Fig. 65); carapace without median furrow; clypeus with distinct median process about $160 \mu \mathrm{m}$ long; sternum wider than long $(0.65 / 0.55)$, unmodified. Chelicerae as in Fig. 74 , with pair of tiny lateral processes in rather distal position, without stridulatory ridges.

PALPS. As in Figs 68-69 and 72-73, coxa unmodified, trochanter with long ventral apophysis and short projection at retrolateral trochanter-femur joint, femur with finger-shaped retrolateral process and large ventral bulge distally, procursus with two membranous ventral processes, one arising from ventral 'knee', the other more distally, bulb with large uncus with flat curved process and bifid appendix, embolus membranous with many fringes distally.

LEGS. Without spines and curved hairs, few vertical hairs; retrolateral trichobothrium on tibia 1 at $3.5 \%$; prolateral trichobothrium absent on tibia 1, present on other tibiae; tarsus 1 with $>30$ indistinct pseudosegments.

VARIATION. Tibia 1 in 3 other males: 7.5, 7.8, 8.0. Sternum variably dark.

\section{Female}

In general similar to male but only weak V-mark on carapace, ocular area and clypeus not darkened, triads not on stalks and closer together (distance PME-PME $240 \mu \mathrm{m}$ ), clypeus unmodified, abdominal marks indistinct. Tibia 1 in 5 females: 6.2-7.1 (mean 6.8). Epigynum weakly sclerotized, anterior plate simple with median incision posteriorly (Figs 67, 70, 75); internal genitalia as in Figs 71, 76.

\section{Natural history}

The spiders were found on the undersides of large leaves, with their bodies flat against the leaf.

\section{Distribution}

Known from type locality only (Fig. 26). 


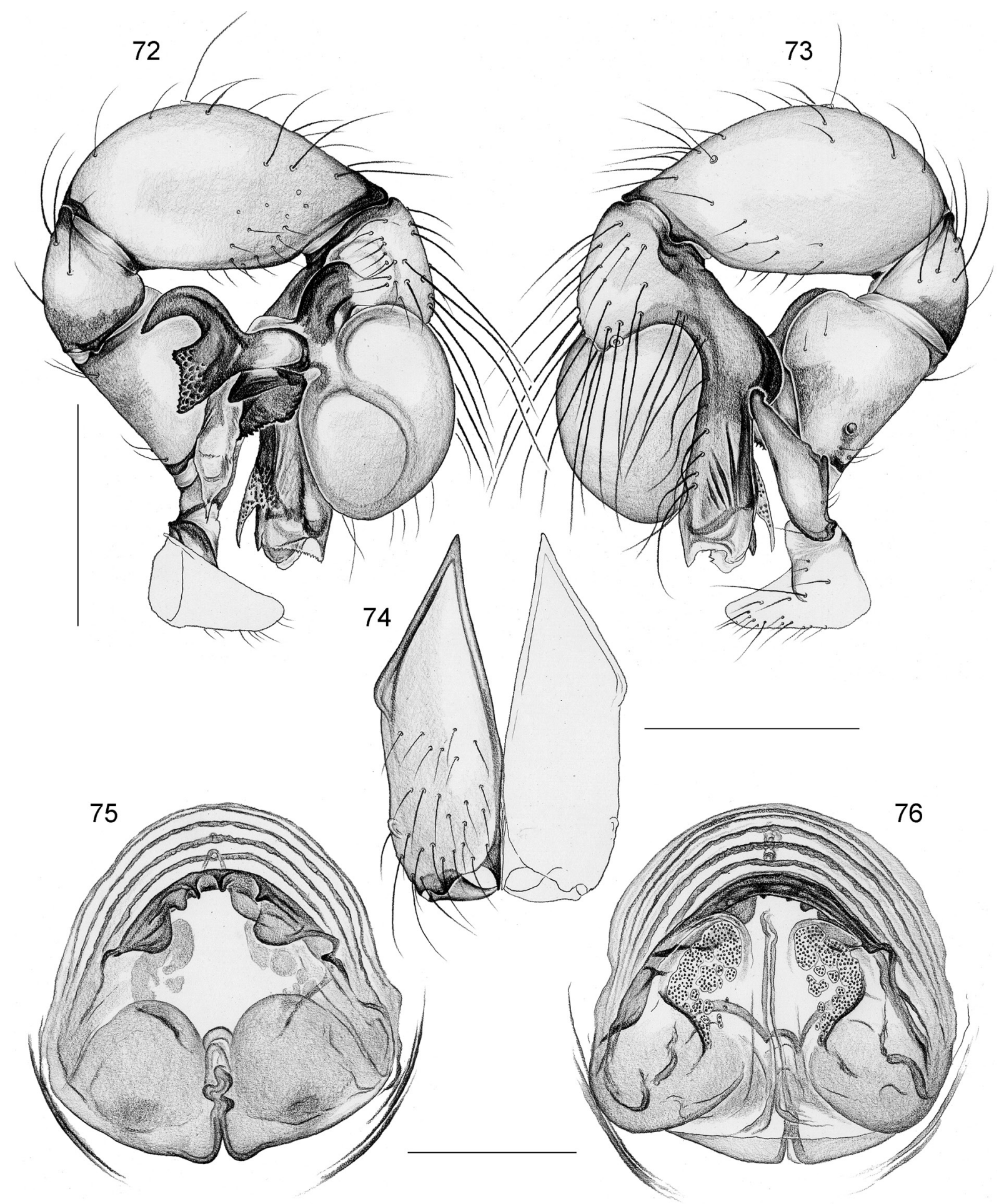

Figs 72-76. Leptopholcus kintampo sp. nov. 72-73. Left $\widehat{o}$ palp, prolateral and retrolateral views. 74. $\hat{\sigma}$ chelicerae, frontal view. 75-76. Cleared + genitalia, ventral and dorsal views. Scale lines: 72-73 = $0.5 \mathrm{~mm} ; 74-76=0.3 \mathrm{~mm}$. 


\section{Spermophora akwamu sp. nov. urn:1sid:zoobank.org:act:D0DB384E-4A3F-4EB1-80D6-48278C31EB82}

Figs 11-13, 34, 77-91

\section{Diagnosis}

Distinguished from the very similar $S$. dieke and from a similar undescribed species from Congo DR (in MRAC) by pair of processes on male clypeus (Fig. 80; only one median process in S. dieke; bifid process in species from Congo DR); from other congeners by armature of male chelicerae (only one pair of weakly sclerotized proximal processes; Fig. 89), by shapes of procursus (ventral sclerotized flap, distal flagellum; Figs 87-88) and bulb (shape of hooked apophysis; Fig. 87), and by shape of epigynum (rectangular plate without pockets; Figs 85, 90).

\section{Etymology}

The name is a noun in apposition, derived from the Akwamu (or Akuambo), an Akan kingdom in the $17^{\text {th }}$ and $18^{\text {th }}$ centuries whose founders settled in the area of modern-day Kakum National Park.

\section{Type material}

Holotype $\widehat{\jmath}$, in ZFMK (Ar 10522).

\section{Type data}

GHANA: Central Region: Kakum National Park $\left(5^{\circ} 20.9^{\prime} \mathrm{N}, 1^{\circ} 23.0^{\prime} \mathrm{W}\right), 160 \mathrm{~m}$ a.s.l., forest near entrance, day collecting, 19 Feb. 2013 (B.A. Huber).

\section{Other material examined}

GHANA: Central Region: 15 $\widehat{\partial} 25$ $q \uparrow 8$ juvs in ZFMK (Ar 10523-24), Kakum National Park, same data as holotype; 7 ठึ 7 우 2 juvs in ZFMK (Ar 10525), same data but 20 Feb. 2013; 1 ठ 8 우 2 juvs

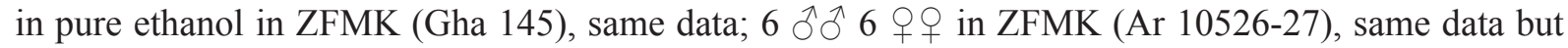
night collecting, 20 Feb. 2013; 11 ऽో fogging in primary forest, 16-25 Nov. 2005 (R. Jocqué, D. De Bakker, L. Baert); 8 $\widehat{\partial} 20$ 우 0 in MRAC (217687, 701, 703, 708), same data but secondary forest, 12-19 Nov. 2005; 1 juv. in MRAC (217292), same data but beating in secondary forest, 23 Nov. 2005; 3 \ठ 2 우 in MRAC $(217261,277)$, same data but beating between primary and secondary forest, 11-12 Nov. 2005; 1 đ 2 우 01 juv. in MRAC (217173), same data but sieving of forest litter, 10 Nov. 2005. Eastern Region: $2 \hat{o} 1 q 1$ juv. in ZFMK (Ar 10528), Atewa Hills, Atewa Atwirebu Reserve at $500 \mathrm{~m}$ a.s.l. $\left(6^{\circ} 13.8^{\prime} \mathrm{N}, 0^{\circ} 32.4^{\prime} \mathrm{W}\right)$, degraded forest along road, 24 Feb. 2013 (B.A. Huber); 1 क 5 juvs in ZFMK (Ar 10529), Atewa Atwirebu Reserve at $740 \mathrm{~m}$ a.s.1. (6 $\left.6^{\circ} 13.8^{\prime} \mathrm{N}, 0^{\circ} 33.5^{\prime} \mathrm{W}\right), 25$ Feb. 2013 (B.A. Huber); 1 juv. in pure ethanol in ZFMK (Gha 139), same data. Western Region: 5 ภิ๊ 4 우 2 juvs in ZFMK (Ar 10530), Ankasa National Park $\left(5^{\circ} 13.0^{\prime} \mathrm{N}, 2^{\circ} 39.1^{\prime} \mathrm{W}\right), 180 \mathrm{~m}$ a.s.l., forest near entrance, day collecting, 22 Feb. 2013 (B.A. Huber);

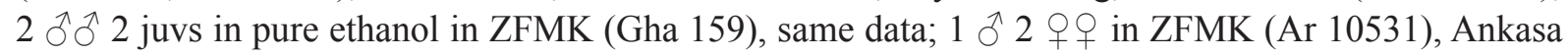
National Park, forest along Big Tree Trail ( $\left.\sim 5^{\circ} 15.1^{\prime} \mathrm{N}, 2^{\circ} 38.4^{\prime} \mathrm{W}\right), \sim 100 \mathrm{~m}$ a.s.1., 23 Feb. 2013 (B.A. Huber).

\section{Description}

\section{Male (holotype)}

Measurements. Total body length 2.1, carapace width 0.7. Leg 1: $22.3(5.3+0.4+5.4+9.2+2.0)$, tibia 2: 3.1, tibia 3: 2.0, tibia 4: 3.0; tibia 1 L/d: 76. Distance PME-PME $115 \mu \mathrm{m}$, diameter PME $95 \mu \mathrm{m}$, distance PME-ALE $35 \mu \mathrm{m}$, no AME. 

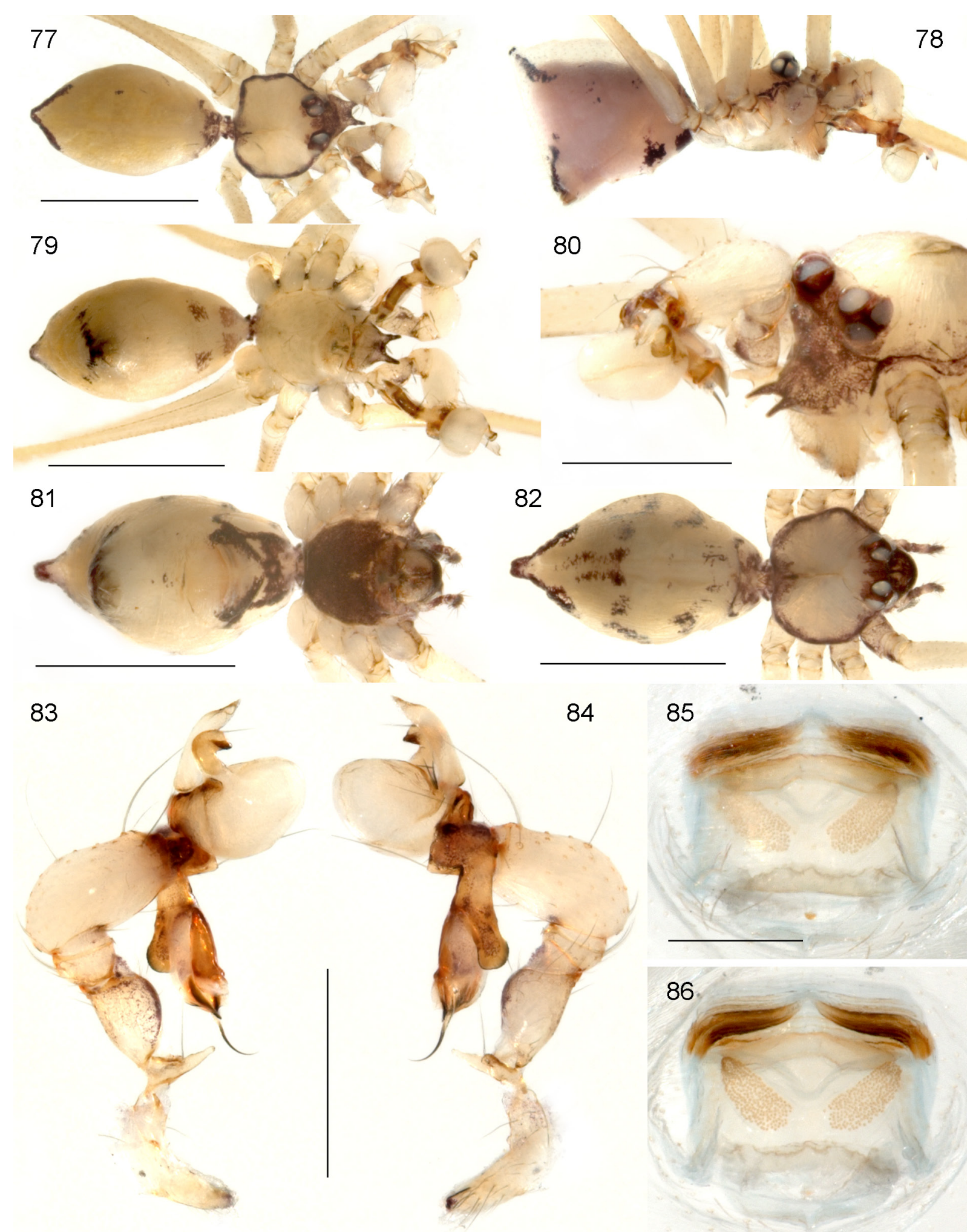

Figs 77-86. Spermophora akwamu sp. nov. 77-79. $\partial$, dorsal, lateral, and ventral views. 80. $\widehat{\partial}$ prosoma, oblique frontal view. 81-82. + , ventral and dorsal views. 83-84. Left $\widehat{o}$ palp, prolateral and retrolateral views. 85-86. Cleared $q$ genitalia, ventral and dorsal views. Scale lines: 77-79, 81-82 $=1 \mathrm{~mm}$; 80, 83$84=0.5 \mathrm{~mm} ; 85-86=0.2 \mathrm{~mm}$. 


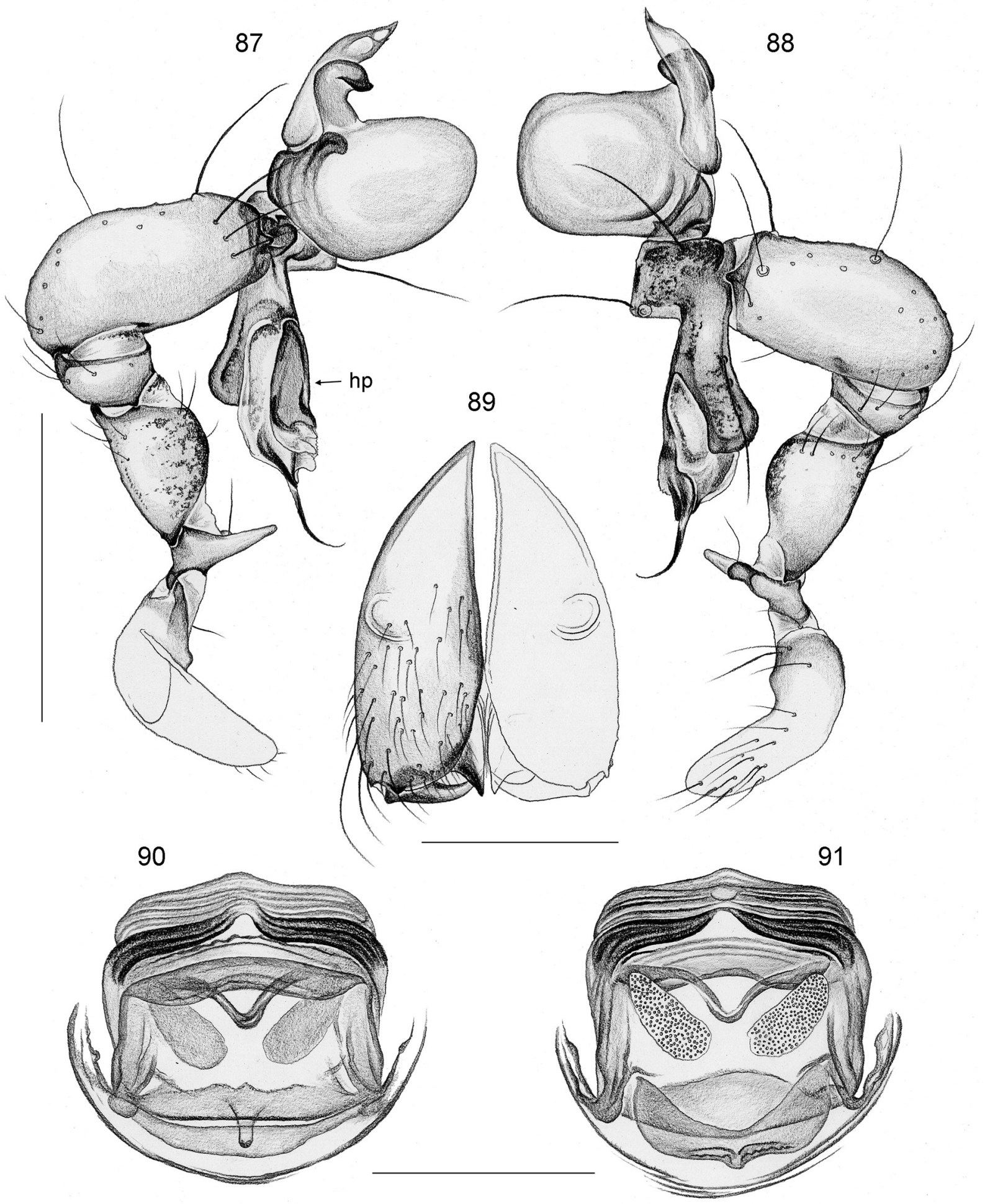

Figs 87-91. Spermophora akwamu sp. nov. 87-88. Left ð palp, prolateral and retrolateral views (hp: hinged process). 89. $\delta$ chelicerae, frontal view. 90-91. Cleared $q$ genitalia, ventral and dorsal views. Scale lines: $87-88=0.5 \mathrm{~mm} ; 90-91=0.3 \mathrm{~mm} ; 89=0.2 \mathrm{~mm}$. 
Color. Carapace pale gray with narrow black margins and indistinct median line (Fig. 77), ocular area and clypeus dark brown to black, sternum pale gray (Fig. 79), legs ochre-yellow, with indistinct dark rings subdistally on femora and tibiae, abdomen mostly pale gray, with black transversal mark at posterior tip, further black marks posteriorly above spinnerets and near gonopore.

Body. Habitus as in Figs 77-79; ocular area slightly elevated, each triad on low hump; carapace without median furrow (only dark line); clypeus with distinctive pair of dark processes, about $100 \mu \mathrm{m}$ long (Fig. 80); sternum wider than long (0.55/0.45), unmodified. Chelicerae as in Fig. 89, with pair of weakly sclerotized processes proximally, distally without modification, without stridulatory ridges.

PALPS. As in Figs 83-84 and 87-88, coxa with indistinct ventral process, trochanter with long ventral and shorter retrolateral apophyses, procursus with ventral sclerotized flap, prolatero-dorsal hinged process and thin distal flagellum; bulb with hooked apophysis and long weakly sclerotized embolus.

Legs. Without spines and curved hairs, few vertical hairs; retrolateral trichobothrium on tibia 1 at $8.5 \%$; prolateral trichobothrium absent on tibia 1, present on other tibiae; tarsus 1 with $>20$ indistinct pseudosegments.

VARIATION. Tibia 1 in 35 other males: 4.3-5.6 (mean 4.8). Some males from type locality with additional small black marks dorsally on abdomen; in both males from Atewa Hills, these marks are present and larger; in males from Ankasa N.P. the abdomen is almost monochromous, without ventral marks, without marks above spinnerets, only mark at posterior tip.

\section{Female}

In general similar to male but sternum in most females dark brown or black (Fig. 81), abdomen dorsally with more and larger black marks (Fig. 82), clypeus unmodified, carapace with small median process acting against indistinct sclerotized plate above pedicel. Tibia 1 in 42 females: 3.5-4.4 (mean 3.9). Epigynum simple rectangular plate, anteriorly with dark transversal internal structure, without pockets (Figs 85, 90); internal genitalia as in Figs 86, 91.

\section{Relationships}

Judging from the very similar morphology, this species is probably closely related to $S$. dieke from Guinea (cf. figures in Huber 2009) as well as to a further very similar (undescribed) species from Congo DR (in MRAC).

\section{Natural history}

The spiders were found both under green leaves where they built slightly domed sheets that extended beyond the leaves (Fig. 13; with the spider resting under a leaf), and in the leaf litter, under curved dead leaves, in small webs apparently not extending beyond the leaf.

\section{Distribution}

Known from several localities in southern Ghana (Fig. 34).

Spermophora ziama sp. nov. urn:1sid:zoobank.org:act:0ACE7F4F-6D69-4F46-A1D3-ED54AAE3846D

Figs 34, 92-105

Spermophora cf. tonkoui - Dimitrov et al. 2013 (DNA data). 


\section{Diagnosis}

Distinguished from S. tonkoui (the only similar species and probably closest known relative; see below) by relatively shorter palpal elements (femur, tibia, bulb; Figs 100-101), by large hooked apophysis on bulb (Fig. 100; in S. tonkoui pair of small apophyses distally on long bulb), by shorter proximal frontal apophyses on male chelicerae (Fig. 103), and by pair of small pockets in posterior epigynal plate (Fig. 104).

\section{Etymology}

The species name is a noun in apposition, derived from the type locality.

\section{Type material}

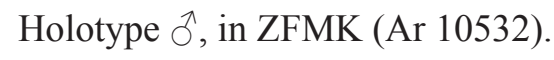

\section{Type data}

GUINEA: Guinée Forestière: Forêt Classée de Ziama $\left(8^{\circ} 24.2^{\prime}\right.$ N, $\left.9^{\circ} 19.3^{\prime} \mathrm{W}\right), 640 \mathrm{~m}$ a.s.1., 2 Dec. 2008 (B.A. Huber).

\section{Other material examined}

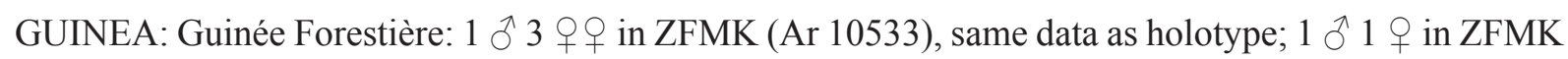
(Ar 10534), same data, taken alive and died from heat; 4 우 1 juv. in pure ethanol in ZFMK (Gui 67), same data.

\section{Description}

\section{Male (holotype)}

Measurements. Total body length 2.0, carapace width 0.9. Leg 1: $19.3(4.6+0.4+5.0+7.1+2.2)$, tibia 2: 3.0, tibia 3: 2.1, tibia 4: 2.8; tibia 1 L/d: 57. Distance PME-PME $345 \mu \mathrm{m}$, diameter PME $70 \mu \mathrm{m}$, distance PME-ALE $35 \mu \mathrm{m}$, no AME.

COLOR. Carapace and clypeus mostly light brown except lighter median band, sternum dark brown, legs ochre-yellow, abdomen mostly dark except dorsal median mark and book-lung covers pale gray.

Body. Habitus as in Figs 92-93; ocular area barely elevated, each triad on short stalk directed laterally, with pair of elongate furrows behind each stalk; carapace without median furrow; clypeus unmodified; sternum wider than long (0.65/0.50), unmodified. Chelicerae as in Figs 102-103, with pair of lateral processes proximally, pair of frontal apophyses proximally close together, and pair of frontal apophyses distally provided with five tiny modified (conical) hairs each; without stridulatory ridges.

PALPS. As in Figs 94-95 and 100-101, coxa unmodified, trochanter with retrolateral apophysis curved towards dorsally, femur with retrolatero-dorsal process proximally, widened distally, tibia very long, dorso-distal rim slightly projecting, procursus complex, apparently with two hinges in proximal part, with long membranous process arising from hinge retrolaterally, distal whitish part provided with many short pseudotrichia ventrally; bulb elongated, with hooked apophysis and long weakly sclerotized embolus.

LEGS. Without spines and curved hairs, few vertical hairs (most hairs missing); retrolateral trichobothrium on tibia 1 at 14\%; prolateral trichobothrium absent on tibia 1, present on other tibiae; tarsus 1 with $>30$ very indistinct pseudosegments.

VARIATION. Tibia 1 in 2 other males: 4.8, 5.4. 


\section{Female}

In general similar to male but triads closer together (distance PME-PME $185 \mu \mathrm{m}$ ), furrows behind ocular area less distinct (Fig. 96). Tibia 1 in 2 females: 4.6 (both). Epigynum large bulging area with median internal tube-like structure, posterior narrow plate with pair of small pockets (Figs 97, 98, 104); internal genitalia as in Figs 99, 105.
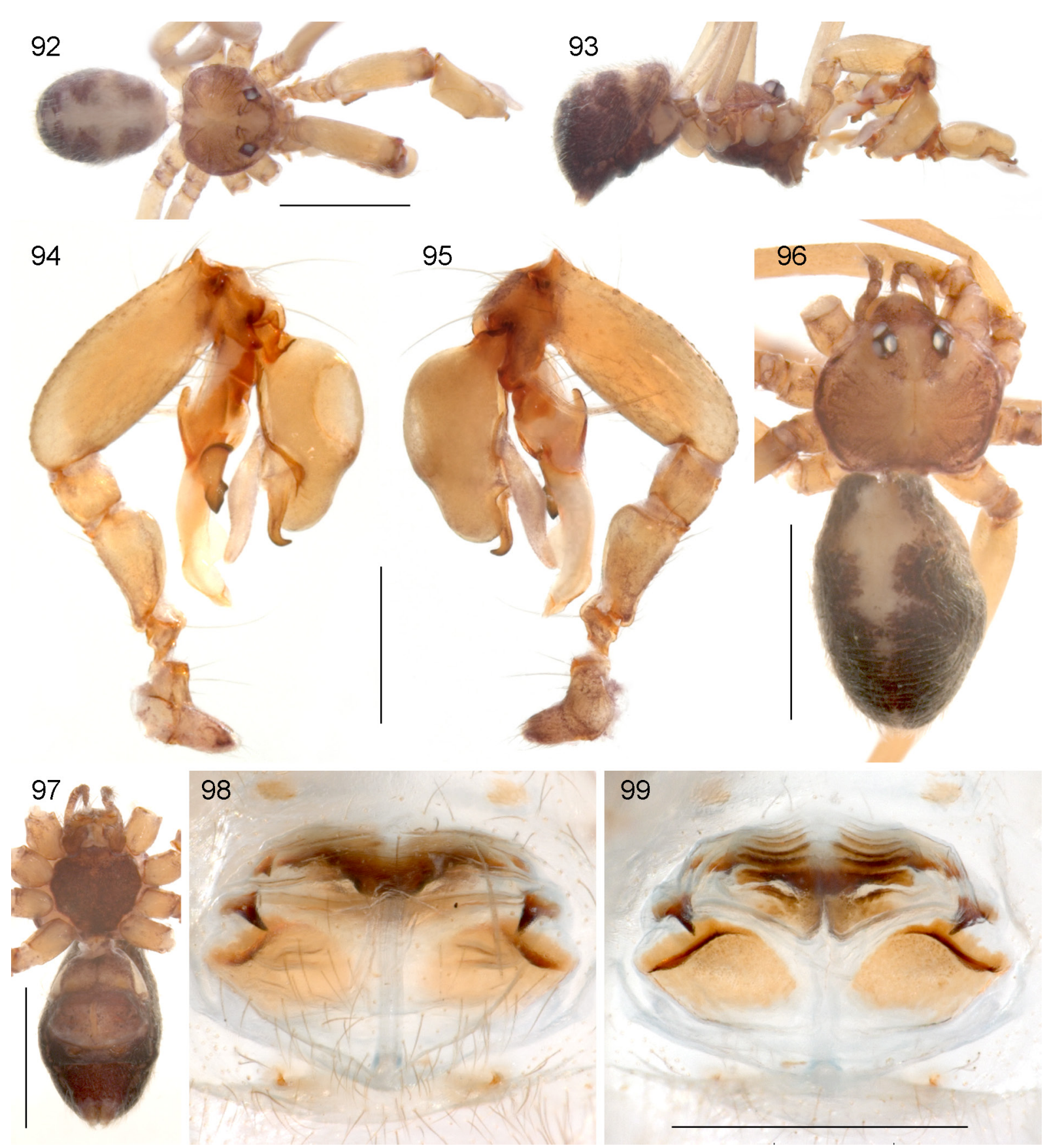

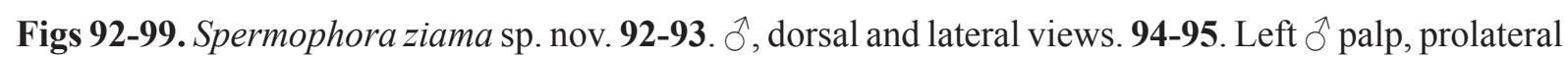
and retrolateral views. 96-97. + , dorsal and ventral views. 98-99. Cleared $q$ genitalia, ventral and dorsal views. Scale lines: 92-93, 96-97 = $1 \mathrm{~mm}$; 94-95, 98-99=0.5 mm. 


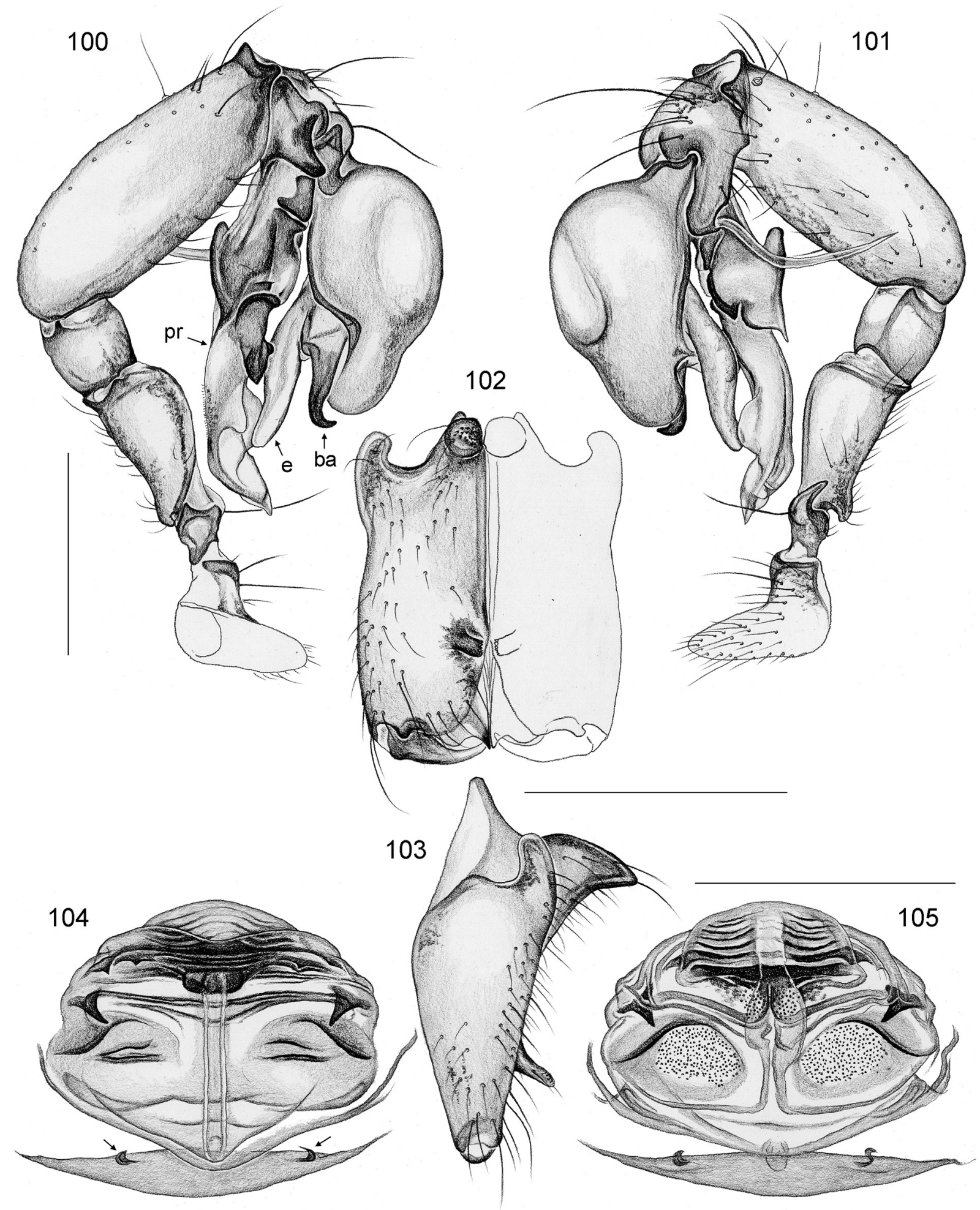

Figs 100-105. Spermophora ziama sp. nov. 100-101. Left $\widehat{\partial}$ palp, prolateral and retrolateral views (ba = bulbal apophysis; $\mathrm{e}=$ embolus; $\mathrm{pr}=$ procursus). 102-103. $\sigma^{\lambda}$ chelicerae, frontal and lateral views. 104-105. Cleared $q$ genitalia, ventral and dorsal views. Arrows point at pockets in posterior epigynal plate. Scale lines: $100-101,104-105=0.5 \mathrm{~mm} ; 102-103=0.3 \mathrm{~mm}$. 


\section{Relationships}

Several unique characters indicate that $S$. ziama sp. nov. is closely related to $S$. tonkoui: curved retrolateral apophysis on male palpal trochanter; procursus with long transparent retrolateral process and distinctive prolateral sclerite between two putative hinges; distal whitish part of procursus with many short pseudotrichia ventrally; male cheliceral armature (proximal frontal apophyses close together); and tube-like internal structure in female genitalia.

The relationships of these two species to other taxa remain obscure. Spermophora tonkoui was explicitly assigned tentatively to the genus (Huber 2003b), and recent analyses of molecular data of S. ziama sp. nov. (Dimitrov et al. 2013: 12S, 18S, h3) provided contradicting results, mostly poorly supported but suggesting an affinity to a group consisting of African Spermophora and the genera Buitinga Huber, 2003, Paramicromerys Millot, 1946, Spermophorides Wunderlich, 1992, and Belisana Thorell, 1898 (Dimitrov et al. 2013: supplement fig. S2).

\section{Natural history}

The spiders were found in crevices in the ground, between soil and roots or between soil and rocks.

\section{Distribution}

Known from type locality only (Fig. 34).

Quamtana kitahurira Huber, 2003

Figs 106-113

Quamtana kitahurira Huber, 2003c: 513, figs 24, 204-208 (ð, Uganda).

Quamtana kitahurira - Huber \& Warui 2012: 12 (ฏ, Rwanda).

\section{New records}

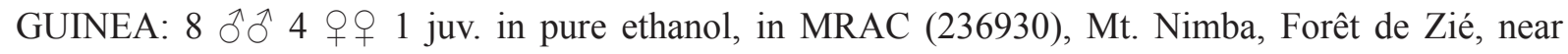
Gouan camp or "Station de Pompage Zié" [740’N, $8^{\circ} 26^{\prime} \mathrm{W}$ ], $1250 \mathrm{~m}$ a.s.l., canopy fogging of trees and understory shrub layer, 3 Oct. 2011 (D. Van den Spiegel, A. Henrard); 1 o in pure ethanol, in MRAC (236929), same data but beating trees and shrubs with hanging litter at 1.5-3 $\mathrm{m}$ above ground.

ANGOLA: $1{ }^{\wedge}$ in SMF (Ang 59.1), near Dundo [ $\left.7^{\circ} 23^{\prime} \mathrm{S}, 20^{\circ} 51^{\prime} \mathrm{E}\right]$, gallery forest of Chilanda river, in bushes, 4 Oct. 1946 (A. de Barros Machado).

\section{Description}

\section{Female}

In general similar to male, habitus as in Figs 106-108; entire animal pale ochre-gray, abdomen light yellow-greenish; tibia 1: 3.4, 3.5, 3.8. Epigynum very simple externally, strongly protruding but weakly sclerotized, with pair of pockets not visible in dissecting microscope (Fig. 110; arrows in Fig. 112); internal genitalia as in Figs 111, 113.

\section{Distribution}

Apparently widely distributed in tropical Africa, but so far only recorded from Uganda, Rwanda, Guinea, and Angola.

\section{Note}

The male from Angola has a slightly more slender procursus but it otherwise identical to specimens from Guinea and East Africa; tibia 1: 4.0. 


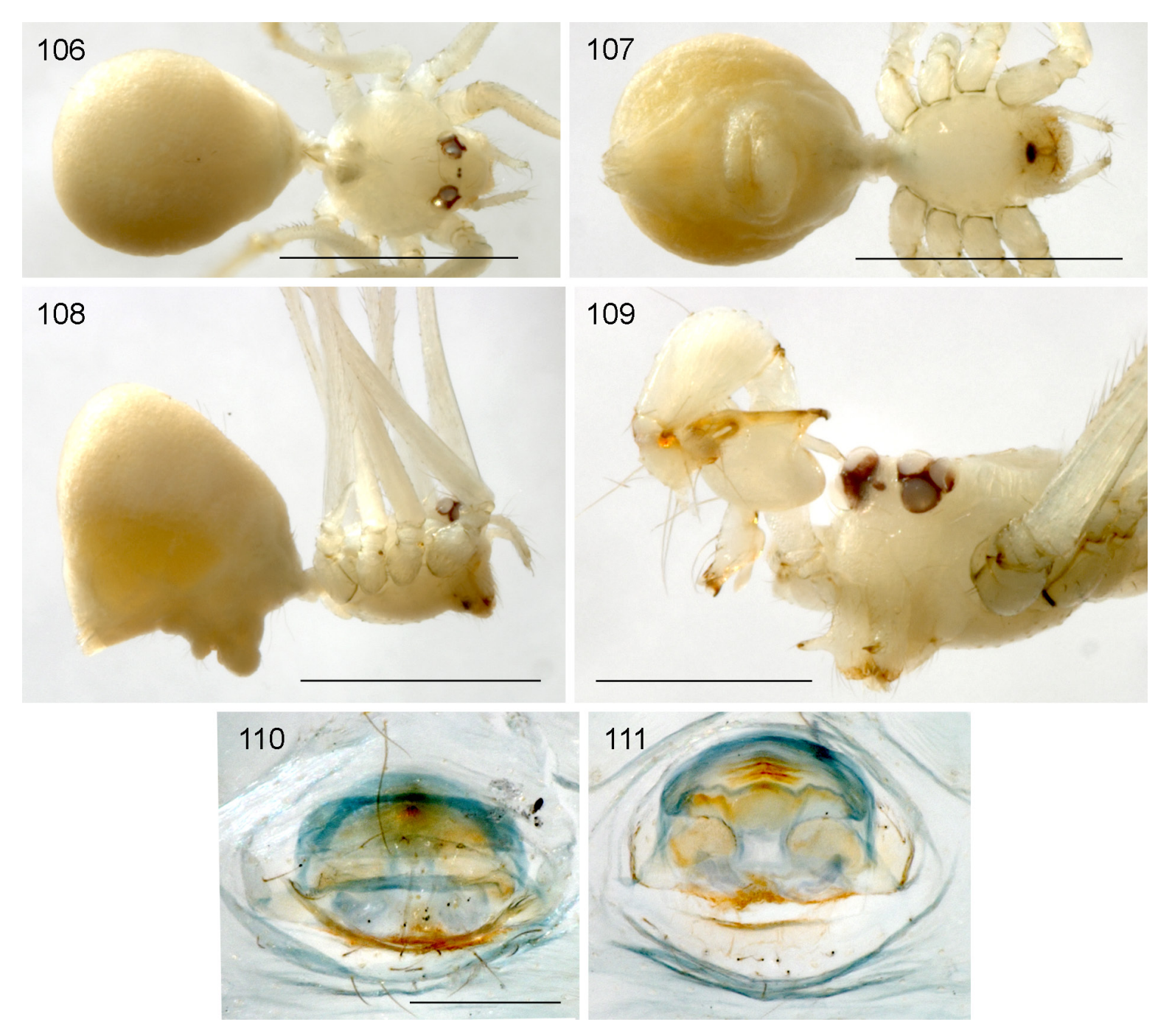

Figs 106-111. Quamtana kitahurira Huber, 2003. 106-108. $q$, dorsal, ventral, and lateral views. 109. $\delta$ prosoma, oblique frontal view. 110-111. Cleared + genitalia, ventral and dorsal views. Scale lines: $106-108=1 \mathrm{~mm} ; 109=0.5 \mathrm{~mm} ; 110-111=0.2 \mathrm{~mm}$.
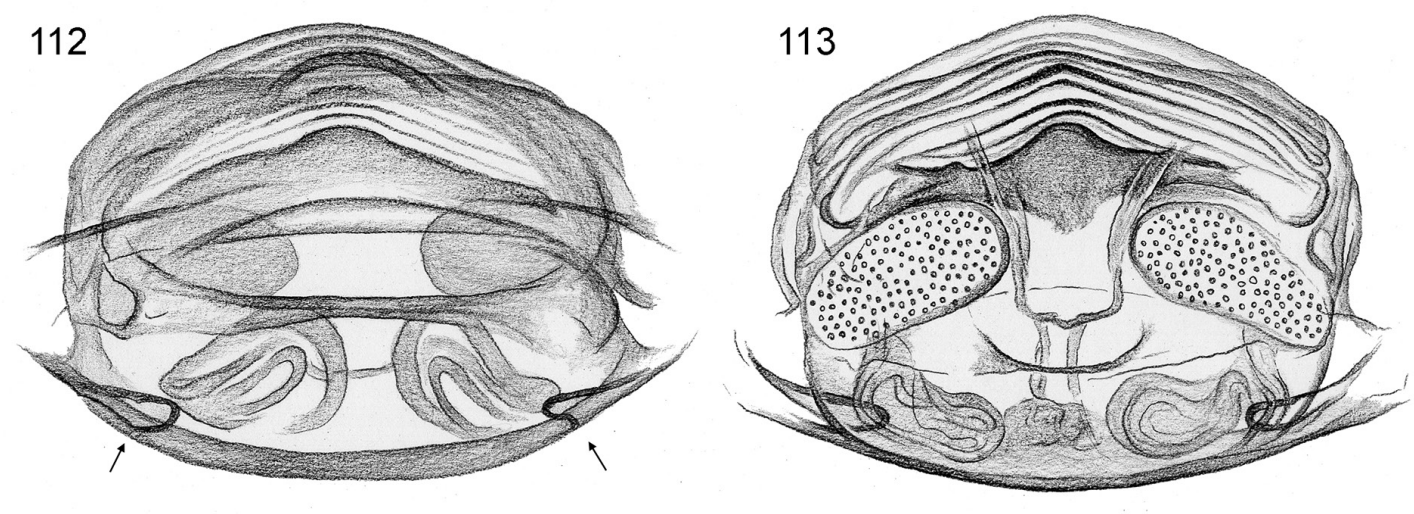

Figs 112-113. Quamtana kitahurira Huber, 2003. Cleared $q$ genitalia, ventral and dorsal views. Arrows point at epigynal pockets. Scale line: $0.2 \mathrm{~mm}$. 


\section{Discussion}

\section{Distribution patterns}

West African pholcids can be divided into five groups (not monophyla) according to their distribution patterns:

\section{West African endemics}

Most species listed above (23, i.e. $61 \%$; or $72 \%$ if introduced species are not counted) are endemics of the Upper Guinean subregion of the Guineo-Congolian center of endemism. About half of these species belong to species groups that are endemic to the Upper Guinean subregion: the Pholcus guineensis group (five species; Huber 2011b); the Smeringopina guineensis and ankasa groups (three species each; Huber 2013); and Spermophora tonkoui + S. ziama sp. nov. Pholcus berlandi from Senegal seems to belong in a species group more diverse in northern Africa (Huber 2011b). All other species belong in tropical African genera and species groups: Anansus is widespread in tropical Africa (Huber 2007; Huber \& Warui 2012), with undescribed species in Ethiopia and Angola (interspecific relationships have not been studied; B.A. Huber unpubl. data); Leptopholcus guineensis and L. kintampo sp. nov. belong in a species group ranging all across tropical Africa (Huber 2011b); Spermophora dieke and S. akwamu sp. nov. belong in a species group that includes an undescribed species from Congo D.R. (B.A. Huber unpubl. data); Smeringopina beninensis is a notable outlier of a group otherwise restricted to Lower Guinea (Huber 2013); finally, Smeringopina fon is a species with unclear relationships (closest relatives in Upper or Lower Guinea; Huber 2013).

\section{Sudanian species}

Artema bunkpurugu sp. nov. and Crossopriza soudanensis are restricted to the northern part of West Africa as defined herein. Both constitute the western limits of genera that are not tropical but range from Sudanian West Africa to Central Asia and northern India (B.A. Huber unpubl. data).

\section{Guineo-Congolian species}

Six species are not restricted to the Upper Guinean subregion but cross the Dahomey Gap (and the Cross River Basin) into Lower Guinea or even into Congolia: Nyikoa limbe (Upper and Lower Guinea; Huber 2007, 2009); Leptopholcus tipula (entire Guineo-Gongolian region from Guinea to Albertine Rift; Huber 2011b); Pholcus kakum which has not yet been found in Lower Guinea but ranges deeply into Congolia (Huber 2011b); Smeringopus cylindrogaster (entire Guineo-Congolian region at least up to Congo River; closest relatives in Lower Guinea and Congolia; Huber 2012); Spermophora kyambura (entire Guineo-Gongolian region from Ghana to Albertine Rift, but note that specimens from Ghana differ slightly from eastern populations; Huber \& Warui 2012); finally, Quamtana kitahurira, which has been collected in montane forests of the Albertine Rift (Uganda, Rwanda) and is here newly recorded from Mt. Nimba in Guinea and northeastern Angola.

\section{Widespread African species}

A single West African species is widespread across several African forest blocks and transition zones: Pehrforsskalia conopyga, which ranges from Cape Verde to southern Africa and the Arabian Peninsula (Huber 2011b), possibly as a result of its tolerance of severely degraded forests. The two congeners known are both East African (Huber 2011b).

\section{Synanthropic species}

Six widespread synanthropic species originated outside West Africa or even outside Africa and have attained pantropical distributions by human transport: Physocyclus globosus (all congeners in Mexico and Central America; Valdez-Mondragón 2010); Modisimus culicinus (all congeners around the Caribbean; Huber et al. 2010); Micropholcus fauroti (origin unclear, possibly Middle East; Huber 2011b); Smeringopus pallidus (African genus; closest relatives apparently in eastern Africa; Huber 
2012); Crossopriza lyoni (closest relatives apparently in Middle East; B.A. Huber unpubl. data); and Artema atlanta (origin unclear, possibly Middle East or Central Asia; B.A. Huber unpubl. data).

\section{Diversity and conservation}

Until recently it was unclear if the relatively low pholcid diversity in West Africa as compared to East and Central Africa was an artifact resulting from taxonomic neglect or a true phenomenon (Huber 2003b). In the meantime, data have accumulated that strongly suggest that the species diversity in West Africa is indeed significantly lower than in areas of comparable size in Central and East Africa. A recent overview of East African pholcids resulted in a total of 87 species (Huber \& Warui 2012), and an upcoming summary paper on Central African pholcids will comprise about 80 species (B.A. Huber unpubl. data). This is in each case more than twice the number reported here for West Africa (38). Six expeditions of comparable duration and collecting effort, two to each region, have resulted in very similar differences among regions: only 28 species in Guinea + Ghana versus 60 species in Gabon + Cameroon and 40 species in Kenya + Uganda. Percentages of undescribed species (as of 2008) collected at these expeditions suggest that the undiscovered diversity is much larger in Central Africa (83\%) than in East Africa (53\%) and West Africa (43\%).

A range of ideas have been proposed to explain why Africa in general appears to be less diverse than South America and tropical Asia (White 2001; Livingstone 2001; Lawson \& Klemens 2001; Antonelli \& Sanmartín 2011), and some of these explanations may also apply to the low pholcid diversity of West Africa compared to Central and East Africa.

1. Central Africa has a much larger area that is wet enough to support rain forest (about $3.1 \times 10^{6} \mathrm{~km}^{2}$ vs. $1.2 \times 10^{6} \mathrm{~km}^{2}$ in West Africa; Naughton-Treves \& Weber 2001).

2. Geologically, West Africa is and has been more homogeneous and stable (especially compared to East Africa), resulting in limited opportunities for speciation (Lawson \& Klemens 2001).

3. Pleistocene dry periods have probably affected West Africa more severely than Central Africa, resulting in fewer and smaller refuges of tropical moist forest (Maley 2001).

Human impact in prehistoric times has also been invoked to explain low African diversity in general (White 2001), but whether this was more severe in West than in Central and East Africa is not known.

Another aspect of West African pholcid distribution is pertinent to conservation issues. There seems to be a tendency for West African endemic species to have on average wider distributions than Central African species. Relatively few species in West Africa seem to be local endemics (e.g., Leptopholcus kintampo sp. nov.; Pholcus doucki; Spermophora ziama sp. nov., S. dieke), while this is common both in Central Africa (e.g., in Smeringopina; Huber 2013) and East Africa (e.g., in Buitinga and Spermophora; Huber 2003b; Huber \& Warui 2012). This certainly requires denser sampling but if it turns out to be true it means that most West African species are not immediately threatened with extinction.

\section{Outlook}

After almost a decade of taxonomic work on African pholcids, a fairly realistic framework is emerging about African pholcid diversity and distribution. All major and most small genera represented in Africa have been revised (Huber 2003a, 2003b, 2003c, 2007, 2009, 2011b, 2012, 2013; Huber \& El Hennawy 2007; Huber \& Warui 2012), and the total number of species has more than tripled from 88 in 2002 to 287. At the level of genera, African pholcids can now be considered relatively well known.

However, the fact that the six expeditions mentioned above resulted in $74 \%$ undescribed species $(87$ of 117 species) clearly demonstrates that much taxonomic work remains to be done. The undiscovered species diversity is still tremendous. Material deposited in major museums has largely been incorporated in the above revisions. This means that if any significant progress is to be made, new collecting is inevitable. In West Africa, the major gaps of knowledge are probably in Liberia and Nigeria. The western 
fauna of the Western Guinean forest zone (west of the Sassandra River, i.e. western Ivory Coast and Liberia) is richer than the eastern fauna (e.g., for mammals; Grubb 2001), but Liberia has essentially seen no arachnologists interested in pholcid spiders. Much the same is true for Nigeria, a country almost four times the size of Ghana but with only four pholcid species recorded for the country (versus 18 for Ghana). Nigeria is particularly interesting for its position between Upper and Lower Guinea and with respect to the border between these subregions of the Guineo-Congolian forests (Maley 2001; Grubb 2001; Dowsett-Lemaire \& Dowsett 2001).

\section{Acknowledgements}

BAH thanks all the people who kindly sent specimens used in this study: J. Coddington, C.E. Griswold, P. Jäger, R. Jocqué, E.-A. Leguin, L. Leibensperger, Yu.M. Marusik, N. Platnick, C. Rollard, A. RussellSmith, S. Tchibozo, and J. Wunderlich. BAH thanks R. Duncan and M. Sidibe for their help in acquiring collection and export permits in Guinea and J. Bosumtwe for his invaluable and friendly assistance during the collecting trip in Ghana. The German Research Foundation provided financial support (DFG, HU 980/9-1).

\section{References}

Antonelli A. \& Sanmartín I. 2011. Why are there so many plant species in the Neotropics? Taxon 60 (2): 403-414.

Deeleman-Reinhold C.L. \& van Harten A. 2001. Description of some interesting, new or little known Pholcidae (Araneae) from Yemen. In: Prakash I. (ed.) Ecology of Desert Environments: 193-207. Scientific Publishers, Jodhpur.

Dimitrov D., Astrin J.J. \& Huber B.A. 2013. Pholcid spider molecular systematics revisited, with new insights into the biogeography and the evolution of the group. Cladistics 29 (2): 132-146. http://dx.doi. org/10.1111/j.1096-0031.2012.00419.x

Dippenaar-Schoeman A.S. \& Jocqué R. 1997. African Spiders: An Identification Manual. Plant Protection Research Institute Handbook 9, ARC-Plant Protection Research Institute, Pretoria.

Dowsett-Lemaire F. \& Dowsett R.J. 2001. African forest birds. In: Weber W., White L.J.T., Vedder A. \& Naughton-Treves L. (eds) African Rain Forest Ecology and Conservation: 233-262. Yale University Press, New Haven and London.

Grubb P. 2001. Endemism in African rain forest mammals. In: Weber W., White L.J.T., Vedder A. \& Naughton-Treves L. (eds) African Rain Forest Ecology and Conservation: 88-100. Yale University Press, New Haven and London.

Huber B.A. 1996. On the distinction between Modisimus and Hedypsilus (Pholcidae; Araneae), with notes on behavior and natural history. Zoologica Scripta 25 (3): 233-240. http://dx.doi. org/10.1111/j.1463-6409.1996.tb00164.x

Huber B.A. 2000. New World pholcid spiders (Araneae: Pholcidae): a revision at generic level. Bulletin of the American Museum of Natural History 254: 1-348. http://dx.doi.org/10.1206/00030090(2000)254<0001:NWPSAP $>2.0 . \mathrm{CO} ; 2$

Huber B.A. 2001. The pholcids of Australia (Araneae; Pholcidae): taxonomy, biogeography, and relationships. Bulletin of the American Museum of Natural History 260: 1-144. http://dx.doi. org/10.1206/0003-0090(2001)260<0001:TPOAAP $>2.0 . \mathrm{CO} ; 2$

Huber B.A. 2003a. Cladistic analysis of Malagasy pholcid spiders reveals generic level endemism: revision of Zatavua n. gen. and Paramicromerys Millot (Pholcidae, Araneae). Zoological Journal of the Linnean Society 137 (2): 261-318. http://dx.doi.org/10.1046/j.1096-3642.2003.00046.x 
Huber B.A. 2003b. High species diversity in one of the dominant groups of spiders in East African montane forests (Araneae: Pholcidae: Buitinga n. gen., Spermophora Hentz). Zoological Journal of the Linnean Society 137 (4): 555-619. http://dx.doi.org/10.1046/j.1096-3642.2003.00053.x

Huber B.A. 2003c. Southern African pholcid spiders: revision and cladistic analysis of Quamtana n. gen. and Spermophora Hentz (Araneae: Pholcidae), with notes on male-female covariation. Zoological Journal of the Linnean Society 139 (4): 477-527. http://dx.doi.org/10.1046/j.0024-4082.2003.00082.x

Huber B.A. 2005. Revision of the genus Spermophora Hentz in Southeast Asia and on the Pacific Islands, with descriptions of three new genera (Araneae: Pholcidae). Zoologische Mededelingen 79-2 (4): 61-172.

Huber B.A. 2007. Two new genera of small, six-eyed pholcid spiders from West Africa, and first record of Spermophorides for mainland Africa (Araneae: Pholcidae). Zootaxa 1635: 23-43.

Huber B.A. 2009. Life on leaves: leaf-dwelling pholcids of Guinea, with emphasis on Crossopriza cylindrogaster Simon, a spider with inverted resting position, pseudo-eyes, lampshade web, and tetrahedral egg-sac (Araneae: Pholcidae). Journal of Natural History 43 (39-40): 2491-2523. http:// dx.doi.org/10.1080/00222930903207876

Huber B.A. 2011a. Phylogeny and classification of Pholcidae (Araneae): an update. Journal of Arachnology 39 (2): 211-222. http://dx.doi.org/10.1636/CA10-57.1

Huber B.A. 2011b. Revision and cladistic analysis of Pholcus and closely related taxa (Araneae, Pholcidae). Bonner zoologische Monographien 58, Zoologisches Forschungsmuseum Alexander Koenig, Bonn.

Huber B.A. 2012. Revision and cladistic analysis of the Afrotropical endemic genus Smeringopus Simon, 1890 (Araneae: Pholcidae). Zootaxa 3461: 1-138.

Huber B.A. 2013. Revision and cladistic analysis of the Guineo-Congolian spider genus Smeringopina Kraus (Araneae, Pholcidae). Zootaxa 3713: 1-160.

Huber B.A. \& Brescovit A.D. 2003. Ibotyporanga Mello-Leitão: tropical spiders in Brazilian semiarid habitats (Araneae: Pholcidae). Insect Systematics and Evolution 34 (1): 15-20. http://dx.doi. org $/ 10.1163 / 187631203788964926$

Huber B.A., Deeleman-Reinhold C. L. \& Pérez G.A. 1999. The spider genus Crossopriza (Araneae, Pholcidae) in the New World. American Museum Novitates 3262: 1-10.

Huber B.A. \& El Hennawy H. 2007. On Old World ninetine spiders (Araneae: Pholcidae), with a new genus and species and the first record for Madagascar. Zootaxa 1635: 45-53.

Huber B.A., Fischer N. \& Astrin J.J. 2010. High level of endemism in Haiti's last remaining forests: a revision of Modisimus (Araneae: Pholcidae) on Hispaniola, using morphology and molecules. Zoological Journal of the Linnean Society 158 (2): 244-299. http://dx.doi.org/10.1111/j.1096-3642.2009.00559.x

Huber B.A., Pérez-González A., Astrin J.J., Blume C. \& Baptista R. 2013. Litoporus iguassuensis Mello-Leitão, 1918 (Araneae, Pholcidae): camouflaged retreat, sexual dimorphism, female color polymorphism, intra-specific genital variation, and description of the male. Zoologischer Anzeiger 252 (4): 511-521. http://dx.doi.org/10.1016/j.jcz.2012.12.001

Huber B.A. \& Warui C.M. 2012. East African pholcid spiders: an overview, with descriptions of eight new species (Araneae, Pholcidae). European Journal of Taxonomy 29: 1-44. http://dx.doi.org/10.5852/ ejt.2012.29

Kraus O. 1957. Araneenstudien 1. Pholcidae (Smeringopodinae, Ninetinae). Senckenbergiana biologica 38 (3/4): 217-243. 
Lawson D.P. \& Klemens M.W. 2001. Herpetofauna of the African rain forest. In: Weber W., White L.J.T., Vedder A. \& Naughton-Treves L. (eds) African Rain Forest Ecology and Conservation: 291-307. Yale University Press, New Haven and London.

Lessert R. de 1938. Araignées du Congo Belge. Revue de Zoologie et de Botanique Africaines 30 (4): 424-457.

Livingstone D.A. 2001. A geological perspective on the conservation of African forests. In: Weber W., White L.J.T., Vedder A. \& Naughton-Treves L. (eds) African Rain Forest Ecology and Conservation: 50-56. Yale University Press, New Haven and London.

Maley J. 2001. The impact of arid phases on the African rain forest through geological history. In: Weber W., White L.J.T., Vedder A. \& Naughton-Treves L. (eds) African Rain Forest Ecology and Conservation: 68-87. Yale University Press, New Haven and London.

Millot J. 1941. Les araignées de l'Afrique occidentale Française. Sicariides et pholcides. Mémoires de l’Académie des sciences de l'Institut de France 64, Académie des sciences, Paris.

Naughton-Treves L. \& Weber W. 2001. Human dimensions of African rain forest. In: Weber W., Naughton-Treves L., Vedder A. \& White L.J.T. (eds) African Rain Forest Ecology and Conservation: 30-43. Yale University Press, New Haven and London.

Platnick N.I. 2013. The world spider catalog, version 13.5. American Museum of Natural History. Available from http://research.amnh.org/iz/spiders/catalog [accessed 30 Aug. 2013]. http://dx.doi. org/10.5531/db.iz.0001

Simon E. 1907. Arachnides recueillis par L. Fea sur la côte occidentale d'Afrique. Annali del Museo civico di storia naturale di Genova 43: 218-323.

Spassky S. 1934. Araneae palaearticae novae. Fam. Pholcidae I. Bulletin du Museum d'histoire naturelle Paris $2^{\text {ème }}$ série 6 (4): 361-372.

Valdez-Mondragón A. 2010. Revisión taxonómica de Physocyclus Simon, 1893 (Araneae: Pholcidae), con la descripción de especies nuevas de México. Revista Iberica de Aracnología 18: 3-80.

Weber W., White L.J.T., Vedder A. \& Naughton-Treves L. (eds) 2001. African Rain Forest Ecology and Conservation. Yale University Press, New Haven and London.

White L.J.T. 2001. The African rain forest. In: Weber W., White L.J.T., Vedder A. \& Naughton-Treves L. (eds.) African Rain Forest Ecology and Conservation: 3-29. Yale University Press, New Haven and London.

Manuscript received: 4 June 2013

Manuscript accepted: 12 September 2013

Published on: 18 October 2013

Topic editor: Rudy Jocqué

Desk editor: Charlotte Thionois

Printed versions of all papers are also deposited in the libraries of the institutes that are members of the EJT consortium: Muséum National d'Histoire Naturelle, Paris, France; National Botanic Garden of Belgium, Meise, Belgium; Royal Museum for Central Africa, Tervuren, Belgium; Natural History Museum, London, United Kingdom; Royal Belgian Institute of Natural Sciences, Brussels, Belgium; Natural History Museum of Denmark, Copenhagen, Denmark. 